\title{
Abstention, ideology and information acquisition ${ }^{1}$
}

\author{
Santiago Oliveros ${ }^{2}$ \\ Royal Holloway, University of London
}

December 8, 2012

\footnotetext{
${ }^{1}$ I thank the editor and two anonymous referees for constructive guidance. I also thank Sophie Bade, Yeon-Koo Che, Ernesto Dal Bo, Rui de Figueiredo, Scott Gelhbach, Hari Govindan, Hugo Hopenhayn, Tim Mylovanov, Philip Neary, Bill Sandholm, Balazs Szentes, Steve Tadelis, Felix Várdy, many classmates and friends, for comments, and Sarah Aiello and Christian Matthew Leister for research assistance. Rody Manuelli, Lucia Quesada and Larry Samuelson provided invaluable guidance, support and help. All errors are mine.

${ }^{2} 215$ McCrae, Department of Economics, Royal Holloway, University of London, Egham Hill, Egham, TW20 0EX. E-mail: Santiago.Oliveros@rhul.ac.uk. Phone: 44 (0) 1784-276506. Fax: 44 (0)1784 439534
} 


\begin{abstract}
We consider an election in which each voter can collect information of different precision. Voters have asymmetric information and preferences that vary both in terms of ideology and intensity. In contrast to all other models of voting with endogenous information, in equilibrium voters collect information of different qualities. We show that information and abstention are not necessarily negatively correlated: some voters are more likely to abstain the more informed they are. We also discuss the manner in which incentives to acquire information are non-monotonic in terms of both ideology and the level of intensity.
\end{abstract}

\title{
JEL Classification:
}

D71, D72, D82.

\section{Keywords:}

Abstention, Information Acquisition, Heterogeneity. 


\section{Introduction}

Very few papers study endogenous information in committees. None of them study abstention or roll-off. Considering that roll-off is usually explained as an informational phenomenon (see [11]), a nexus between information acquisition and abstention seems appropriate to fully understand the decision to vote or not. In this paper we study that nexus and answer the question, who abstains in equilibrium?

We start with a traditional common values model of costless voting where voters' preferences are diverse and private. Our set-up is based on [1]: there is an election between two candidates and voters' preferences depend on two different states of nature. In one state one candidate is preferred to the other candidate by all voters but in the other state the latter candidate is preferred to the former by all voters. Hence voters want to elect the "correct" candidate in each state of nature (in this case they suffer no utility losses) but differ on the utility losses they suffer for mistaken decisions. Voters do not know the true state of nature. Our main innovation is to allow voters to select the quality of a binary signal that is correlated with the true state of nature. We assume that more informative signals entail a higher cost.

We also introduce a richer set of preferences. Traditionally, preferences in committees are modeled with a single parameter that captures the ideological bias. This parameter is sufficient to understand the incentives to vote with exogenous information. But, since the incentives to acquire information depend on the absolute level of utility losses, restricting preferences to abstract from variation in intensity is not without loss of generality. In fact, as we show in this paper, it matters for the study of the link between costly information acquisition and abstention. In contrast to all other papers on voting with endogenous information, voters collect information of different quality in our model, and we show that it is not necessarily true that better informed voters abstain less often.

The existence of an equilibrium with voters endogenously collecting information of different qualities does not follow from a straightforward application of fixed-point arguments. 
We resolve this difficulty by transforming the problem of finding a fixed point in the space of strategies to that of finding a fixed point in the space of "pivotal" probabilities. We then characterize the equilibrium and proceed to study the voter's behavior and, in particular, the connection between information and abstention.

First, rational ignorance (making decisions without acquiring information) is driven by two different forces in our model. On one hand, there are voters with extreme ideology that remain uninformed and vote accordingly that ideology. We call this group of voters "strong supporters". On the other hand, there are some centrists voters with low intensity that decide not to collect information and abstain. We call this group of voters "abstainers". Second, in equilibrium, abstention takes two different forms. On one hand, the previously mentioned rationally ignorant voters with low intensity abstain. On the other hand, some voters collect information and vote if the information reinforces their bias, but abstain if the information goes against their bias. We call these voters "weak supporters". In our model the swing voter's curse happens for two reasons. In one case a centrist voter decides to remain uninformed (see [11]), and in the other case, one of the signals contradicts the mild ideological bias of a non centrist voter (see [7]).

Our model allows us to study in detail the correlation between information and abstention. In particular we can answer the question; do marginally better informed voters abstain with lower probability than marginally less informed voters? While the question of whether informed voters show up more often than uninformed voters may be answered positively at the aggregate level, the effect of being marginally more informed depends on the voter's ideology. We show that, conditional on the state, weak supporters of the correct candidate are more likely to vote the more informed they are but weak supporters of the other candidate are more likely to abstain the more informed they are. The intuition behind these results is related to the combination of ideology and information. Even though more information always implies that the realization of the signal favoring the correct candidate is more likely than a realization against it, the impact of this signal on voting behavior depends on the 
particular voter's ideology. While among weak supporters of the correct candidate this signal is more likely to confirm their ideological bias, among weak supporters of the other candidate this signal is more likely to contradict their ideological bias. To our knowledge, this is the first paper to study how ideology and intensity of preferences interact to affect the relation between (endogenous) information and abstention.

The rest of the paper is organized as follows. After reviewing the literature in the next section we present our model in Section 3. Section 4 presents the existence result and a partial but detailed characterization. In Section 5 we discuss the importance of our assumptions about preferences and we use the model to contribute to some empirical debates relating fundamental voters' characteristics and voting behavior. In particular we apply our model to shed light on the relation between education and turnout and how partisan support affects information acquisition and abstention. All proofs are relegated to an Appendix.

\section{Literature Review}

Our paper is related to voting models with endogenous information ${ }^{1}$ and non-costly voting models of abstention. We discuss each branch of the literature below.

[11] is the first paper providing an explanation for selective abstention or roll-off based on the level of information that a voter receives exogenously. They argue that abstention is a type of delegation that occurs when a voter is poorly informed and suffers the swing voter's curse. $^{2}$ [12] extend their results by introducing heterogeneity both in preferences and in quality of information. They provide examples where "individuals with better information are more likely to participate than individuals with worse information..." ([12], page 382):

\footnotetext{
${ }^{1}[17]$ surveys models with information acquisition in committees.

${ }^{2}$ Abstention has also been studied as a decision theoretic problem as in [19] and [21]. [7] assume that voters abstain because they do not gain much by switching the winner (indifference) or they do not win much by selecting any winner (alienation). [33] allows voters to signal by abstaining, in order to affect the outcome of a second election. [2] provide evidence that the strategic behavior that leads to the swing voter's curse can be replicated in the lab.
} 
"because uninformed independents abstain and informed independents vote, the model provides an informational explanation for why better educated individuals are more likely to vote" ([10], page 104). Both [11] and [12] place the emphasis on differential information. ${ }^{3}$ We show that when information is endogenous, the link between information and abstention is more intricate and requires further consideration of the role of the voter's ideological bias.

The existing literature on committee decision making with endogenous information studies cases where voters collect the same level of information in equilibrium. ${ }^{4}$ [24], [31], [13], [26], [16], [4] and [18] assume that voters are homogenous (at least those willing to collect information) and/or that each voter can receive an independent draw from a common distribution. [25] allows for heterogeneity and the option of acquiring information of different quality, but restricts the environment so that, in equilibrium, every informed voter has the same incentives to collect information. None of these papers study abstention.

There is some evidence that information and turnout are related. [34] use survey and aggregate data on Presidential and House races on the same ballot to show that information and turnout are positively correlated. [6] use data from the National Research Council regarding the quality of different research programs and find that roll-off can be explained by lack of information. [21] and [23] provide evidence that information and turnout are positively correlated correcting for endogeneity of information. Assuming a very particular structure of preferences, [27] finds that more informed voters are more likely to vote. On the other hand [15] finds that more television exposure reduces turnout. He argues that the correlation between information and turnout may still be positive since voters also stopped consulting newspapers and magazines inducing a lower consumption of overall information. In this paper we provide a theoretical argument for the negative correlation between information and abstention.

\footnotetext{
${ }^{3}[28]$ also extends [11] by allowing for exogenous differential quality of information but restricting preference diversity significantly.

${ }^{4}$ The only exception is an example in [24] with a very particular type of heterogeneity in a two-member committee.
} 
We argue that information may or may not be positively correlated with turnout at the individual level. The previous empirical studies could not have found this correlation because they compared aggregate measures without conditioning for ideology at the individual level (which matters, as we demonstrate), or because they define information in a coarse way. [34], [21], and [23] compare informed voters with uninformed voters; and [6] use three different levels of information quality to classify between informed and uninformed. [27] does not control for endogenous information and uses a pure common value set up in a structural model assuming away differences in ideology. All of these strategies lead to testing the composition of the electorate as a whole without considering the individual voter's behavior. To our knowledge, the closest test regarding the effect of marginal changes in information was conducted by [30]. They found that "[in the distance utility model]...the probability of voting for Reagan increases with information level. The opposite is true for Carter." ([30], page 526). As it will become clear, their finding is consistent with our model.

\section{The model}

There is a set $\mathcal{N}$ of potential voters with cardinality $n$ who must decide between two options, $A$ and $Q$. There are two equally likely states of nature $\omega \in\{a, q\}$. The winner is selected according to the plurality rule, and if there is a tie the winner is selected by tossing a fair coin. The set of possible actions for a voter is $\{Q, \emptyset, A\}$ where $Q(A)$ is a vote for candidate $Q(A)$ and $\emptyset$ stands for abstention.

There are two classes of voters: partisan and non-partisan. With probability $\alpha \in(0,1)$ a voter $i$ is partisan. Partisan voters are described in terms of their behavior: with probability $\xi_{x} \in(0,1)$, where $\sum_{x \in\{Q, \emptyset, A\}} \xi_{x}=1$, a partisan voter of type $x \in\{Q, \emptyset, A\}$ casts a ballot $x$. Note that partisans not only support each candidate with some probability but also they abstain with some probability. Non partisan voters have state contingent preferences described by $\theta=\left\{\theta_{q}, \theta_{a}\right\} \in[0,1]^{2}:$ if $A$ is elected in state $q$, then the voter type $\theta=\left\{\theta_{q}, \theta_{a}\right\}$ suffers 
a utility loss of $\theta_{q}$ while if $Q$ is elected in state $a$ she suffers a utility loss of $\theta_{a}$. There is no utility loss for selecting $A$ in state $a$ or $Q$ in state $q$. Voters' preferences are private information. If voter $i$ is non-partisan her preferences are conditionally independently drawn from a distribution with a cumulative distribution function $F$ on $[0,1]^{2}$ with no mass points. We assume further that no hyperplane of $F$ has positive measure (hyperdiffuse distribution) so if we let $g\left(\theta_{a}\right)$ be any function we have that $\int d F\left(\theta_{a}, g\left(\theta_{a}\right)\right)=0 .{ }^{5}$ We assume that $F$, $\alpha$ and $\left\{\xi_{Q}, \xi_{\emptyset}, \xi_{A}\right\}$ are common knowledge. We refer to non partisan voter $i$ 's preferences as her type, and to a "non-partisan voter type $\theta$ " simply as a "type $\theta$ ".

After types are privately revealed, a voter can select the precision $p \in\left[\frac{1}{2}, 1\right]$ of the information she will receive. Where $p$ is the parameter of a Bernoulli random variable $S$ taking values on the set $\left\{s_{q}, s_{a}\right\}$. We assume that signals have the same precision in both states: $p=\operatorname{Pr}\left(s_{\omega} \mid p, \omega\right)$ for $\omega \in\{a, q\}$. Precision is costly and the precision cost is given by $C:\left[\frac{1}{2}, 1\right] \rightarrow \mathcal{R}_{+}$, where we assume that:

Assumption 1 The cost function $C$ is twice continuously differentiable everywhere in $\left[\frac{1}{2}, 1\right]$ and satisfies 1) $C^{\prime}(p)>0$ and $C^{\prime \prime}(p)>0$ for all $p>\frac{1}{2}$, 2) $\left.C^{\prime \prime}\left(\frac{1}{2}\right) \geq C\left(\frac{1}{2}\right)=C^{\prime}\left(\frac{1}{2}\right)=0,3\right)$ $\lim _{p \rightarrow 1} C^{\prime}(p) \rightarrow \infty$

The set of voters $(\mathcal{N})$, the (common) distribution that characterizes these voters' preferences $\left(\alpha, \xi_{Q}, \xi_{\emptyset}, \xi_{A}, F\right)$, and the cost of information function $(C)$, constitute a committee. Since non-partisan voters decide on the precision of their signal and on how they vote after receiving the signal, voter $i$ 's pure strategy consist of an investment function $P^{i}:[0,1]^{2} \rightarrow\left[\frac{1}{2}, 1\right]$ and a voting function $V^{i}:[0,1]^{2} \times\left\{s_{q}, s_{a}\right\} \rightarrow\{Q, \emptyset, A\}$, such that $P^{i}(\theta)$ is the investment level of non partisan voter $i$ with type $\theta$, and $V^{i}(\theta)=\left(V^{i}\left(\theta, s_{q}\right), V^{i}\left(\theta, s_{a}\right)\right)$ is the ballot cast by $i$ upon receiving the signal $s .{ }^{6}$ When we refer to a generic voting function, investment function or strategy, we omit the superscripts. We say that voter $i$ of type $\theta$ uses an informative voting function if and only if $V^{i}\left(\theta, s_{q}\right) \neq V^{i}\left(\theta, s_{a}\right)$. Else, the voter is said

\footnotetext{
${ }^{5}$ We can ignore voters who are indifferent between strategies, as in [5].

${ }^{6} V(\theta)$ describes the voter's behavior and $\left(v_{q}, v_{a}\right) \in\{Q, \emptyset, A\}^{2}$ denotes arbitrary voting functions: vote $v_{q}$ after receiving $s_{q}$ and vote $v_{a}$ after receiving $s_{a}$. We use $v$ to refer to a particular vote.
} 
to use an uninformative voting function. We will identify strategies by their voting function, implicitly assuming that the precision is optimally selected. Since precision is costly if voter $i$ decides to use an uninformative strategy she selects $P^{i}(\theta)=\frac{1}{2}$.

The timing of the game is as follows: 1) Nature draws the profile of types as well as the state, 2) each voter $i$ observes her own preferences, 3) non partisan voter $i$ privately decides whether or not to acquire information by selecting $\left.p^{i} \in\left[\frac{1}{2}, 1\right], 4\right)$ each voter draws a private signal from the selected distribution parameterized by $\left.p^{i}, 5\right)$ voters cast their ballots simultaneously and, 6) the winner is elected according to the plurality rule with ties broken by a fair coin toss.

When voter $i$ decides how to vote she considers her effect on the outcome of the election so she must figure out the probability distribution of the different vote counts among the remaining voters. In order to calculate this probability distribution voter $i$ needs to consider the probability that a random voter casts a vote in favor of one or the other candidate. Focusing on symmetric equilibria we have that the probability that an arbitrary voter $j \neq i$ votes $v$, in state $\omega$, when all players but $i$ are using the strategy $(P(\theta), V(\theta))$ is

$$
\operatorname{Pr}(v \mid \omega)=\alpha \xi_{v}+(1-\alpha) \int_{\theta \in[0,1]^{2}} \sum_{s \in\left\{s_{q}, s_{a}\right\}} \mathbf{I}_{V(\theta, s)=v} \operatorname{Pr}(s \mid P(\theta), \omega) d F(\theta)
$$

where $\mathbf{I}_{x=y}=1$ iff $x=y$ and 0 otherwise. The first part of the right hand side is the probability that a voter is partisan, multiplied by the probability that a partisan voter casts a ballot $v$. The second part is merely the probability that a voter is non partisan multiplied by the probability that a non partisan voter casts the ballot $v$.

Let $\neg(\omega)$ be equal to $A$ when the state is $q$ and equal to $Q$ when the state is $a$; we denote the probability that the outcome is $\neg(\omega)$ in state $\omega$ when voter $i$ casts the vote $v$ as $\operatorname{Pr}(\neg(\omega) \mid \omega, v)$. With this in mind we have the expected utility of voter $i$ that casts a 
ballot $v_{x}$ after receiving the signal $s_{x}$ with precision $p$ is given by

$$
U^{i}\left(p, v_{x} \mid \theta, s_{x}\right)=-\sum_{\omega \in\{q, a\}} \theta_{\omega} \times \operatorname{Pr}((\neg \omega) \mid \omega, v) \times \operatorname{Pr}\left(\omega \mid s_{x}, p\right)
$$

where $\operatorname{Pr}\left(\omega \mid s_{x}, p\right)$ is the posterior beliefs about that state $\omega$ after the signal $s_{x}$ with precision $p$. Note that (2) is related to the incentives to vote after voter $i$ has received the signal but the decision to collect information depends also on the frequency with which these signals are generated by the choice of $p$. Let $\left.\mathcal{U}^{i}\left(p,\left(v_{q}, v_{a}\right)\right) \mid \theta\right)$ be the the gross expected utility of player $i$ of type $\theta$ and investment choice $p$, for a voting strategy $\left(v_{q}, v_{a}\right)$ :

$$
\left.\mathcal{U}^{i}\left(p,\left(v_{q}, v_{a}\right)\right) \mid \theta\right) \equiv \sum_{x \in\{q, a\}} U^{i}\left(p, v_{x} \mid \theta, s_{x}\right) \operatorname{Pr}\left(s_{x}\right)
$$

We study Bayesian equilibria in symmetric profiles of pure strategies.

Definition 1 A symmetric Bayesian equilibrium for the voting game is a strategy $\left(P^{*}(\theta), V^{*}(\theta)\right)$ such that: 1) all voters have the same voting and investment functions: $V^{j}(\theta)=V^{*}(\theta)$ and $P^{j}(\theta)=P^{*}(\theta)$ for every $\theta$, 2) no type $\theta$ has a profitable deviation from $\left(P^{*}(\theta), V^{*}(\theta)\right)$ so ${ }^{7}$

$$
\mathcal{U}^{i}\left(P^{*}(\theta), V^{*}(\theta) \mid \theta\right)-C\left(P^{*}(\theta)\right) \geq \mathcal{U}^{i}\left(p,\left(v_{q}, v_{a}\right) \mid \theta\right)-C(p)
$$

Although we omit other players' strategies in definitions $\operatorname{Pr}(\neg(\omega) \mid \omega, v), U^{i}\left(p, v_{x} \mid \theta, s_{x}\right)$ and $\left.\mathcal{U}^{i}\left(p,\left(v_{q}, v_{a}\right)\right) \mid \theta\right)$, the reader should understand that player $i$ 's payoffs depend on the strategies used by other players.

\footnotetext{
${ }^{7}$ It is well known in statistical decision theory (see [8], page 139) that for a fixed information level the decision problem is separable in the signals. Hence, any strategy that verifies (4) also verifies that $U^{i}\left(P^{*}(\theta), V^{*}(\theta, s) \mid \theta, s\right) \geq U^{i}\left(P^{*}(\theta), v^{\prime} \mid \theta, s\right)$ for each $s \in\left\{s_{a}, s_{q}\right\}$.
} 


\section{Solving the Model}

Because the best responses are complex objects, to solve the model we must take an indirect approach. We first study voter $i$ 's incentives to vote in favor of one or the other candidate in section 4.1. This allows us to identify which strategies might be optimal. In the process we discuss the incentives to abstain and how the swing voter's curse emerges in our model. Once we have identified which strategies can be optimal in equilibrium we proceed to determine which strategy each type selects. For that we start by determining the maximum expected utility that can be generated by a particular voting strategy. For those that involve no information acquisition the problem is easy but for the informative voting strategies we have to study how voters select the precision of the signal. That is the focus of section 4.2.

When studying the information acquisition function we show that the incentives to collect information depend on the level of the pivotal probabilities while the incentives to vote (after receiving the signal) depend only on the ratio of pivotal probabilities. We also discuss how the possibility of abstaining changes the value of information and, hence, makes the information acquisition not continuous. This discontinuity is the source of the problem to prove existence which we solve in subsection 4.3. In that section we also present a partial, but detailed, characterization of equilibrium. Essentially this characterization amounts to determine which types use which strategy. The characterization is partial because it is presented in terms of the probability of the relevant pivotal events which are endogenously determined. Despite this drawback it is detailed enough to allow us to derive interesting conclusions that we present in the end of that subsection and in Section 5 .

\subsection{Voting Incentives}

In order for voter $i$ to be willing to vote $v$ after a signal $s$ she needs to compare the expected utility for this vote, $U^{i}\left(P^{*}(\theta), v \mid \theta, s\right)$, with the induced expected utility for voting any other way $v^{\prime}, U^{i}\left(P^{*}(\theta), v^{\prime} \mid \theta, s\right)$. Because non-partisan voters are rational they condition 
their voting choices on the event in which they are pivotal. When abstention is possible there are many events that lead to the voter being pivotal in some sense. For example, when the voter is considering a vote in favor of $A$ she must focus on the events in which $Q$ is winning by one vote (because voting $A$ creates a tie) or there is a tie (because voting for $A$ makes $A$ the winner). Note that by creating a tie she only changes the winner from $Q$ to $A 50 \%$ of the time and when breaking a tie she only increases $A$ 's chances with respect to the original tie by $50 \%$. Since these events have different occurrence depending on the actual state of nature we denote the probability that the voter actually makes $A$ the winner by voting for $A$ as $\operatorname{Pr}\left(p i v_{A} \mid \omega\right)$. When comparing whether to vote for $A$ or abstaining this is the relevant pivotal event that the voter focus on. Similarly, when comparing a vote for $Q$ with abstention the relevant event is $A$ winning by one vote or a tie, and we denote the probability of this event by $\operatorname{Pr}\left(\operatorname{piv}_{Q} \mid \omega\right)$.

Note that we have focused on comparing a positive vote with the option of abstaining. When comparing two positive votes, a vote for $A$ with a vote for $Q$, there are more situations that are relevant for this voter and it is easy to understand which are these events by performing a double comparison. For a voter to be willing to vote for $A$ instead of $Q$ it must be that $Q$ leads to a lower expected utility than $\emptyset$ and, at the same time, $\emptyset$ leads to a lower expected utility than supporting $A$. Hence, we have that the probability of the relevant pivotal events in comparing a vote for $A$ with a vote for $Q$ is the sum of the probabilities of the event in which a vote for $A$ makes $A$ the winner and the event in which a vote for $Q$ makes $Q$ the winner which we denote $\operatorname{Pr}\left(\operatorname{piv}_{Q} \cup \operatorname{piv}_{A} \mid \omega\right)$.

Now that we have described the relevant pivotal events we focus on identifying when a voter votes for $A, Q$ or abstains. The presence of partisan voters guarantees that all pivotal events occur with at least some probability. Using the definition of expected utility after a particular signal (2) a necessary condition for a non-partisan voter type $\theta$ to vote for $A$ after 
receiving the signal $s$ is

$$
\frac{\theta_{q}}{\theta_{a}} \frac{\operatorname{Pr}(q \mid s, p)}{\operatorname{Pr}(a \mid s, p)} \leq \min \left\{L_{Q \rightarrow A}, L_{\emptyset \rightarrow A}\right\}
$$

where $L_{Q \rightarrow A}=\frac{\operatorname{Pr}\left(p i v_{Q} \cup p i v_{A} \mid a\right)}{\operatorname{Pr}\left(p i v_{Q} \cup p i v_{A} \mid q\right)}$ and $L_{\emptyset \rightarrow A}=\frac{\operatorname{Pr}\left(p i v_{A} \mid a\right)}{\operatorname{Pr}\left(p i v_{A} \mid q\right)}$ are the relative probabilities of each of the relevant pivotal events when comparing the different voting options. A strict inequality gives sufficient conditions. Let's try to understand (5) focusing on the case where $L_{Q \rightarrow A}<L_{\emptyset \rightarrow A}$ so (5) is equivalent to

$$
\theta_{q} \times \operatorname{Pr}\left(p_{i v_{Q}} \cup p i v_{A}, q \mid s, p\right) \leq \theta_{a} \times \operatorname{Pr}\left(p_{i v_{Q}} \cup p i v_{A}, a \mid s, p\right)
$$

The left hand side is divided in two parts: $\theta_{q}$ is the loss incurred for wrongly electing $A$ in state $q$, and $\operatorname{Pr}\left(\operatorname{piv}_{Q} \cup p i v_{A}, q \mid s, p\right)$ is the probability of electing $A$ in state $q$ when the voter votes for $A$ instead of $Q$. Then, the left hand side is the cost of switching a vote from $Q$ to $A$. The right hand side, on the other hand, is the expected benefits for switching a vote from $Q$ to $A$. Hence (5) with $L_{Q \rightarrow A}$ simply states that the expected benefits generated by switching

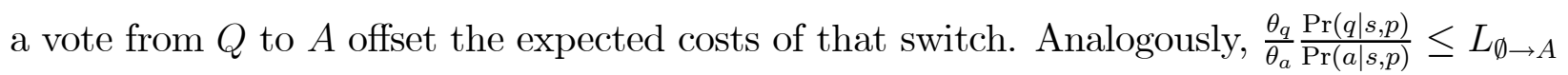
implies that the savings generated by switching from abstention to supporting $A$ offsets the expected costs of that switch. Putting all together (5) simply states that switching a vote from $Q$ to $A$ and from $\emptyset$ to $A$ generates more expected benefits than costs and, hence, a vote for $A$ is preferred than a vote for $Q$ or abstaining.

Analogously a necessary condition for a voter to cast a ballot in favor of $Q$ is

$$
\frac{\theta_{q}}{\theta_{a}} \frac{\operatorname{Pr}(q \mid s, p)}{\operatorname{Pr}(a \mid s, p)} \geq \max \left\{L_{Q \rightarrow A}, L_{Q \rightarrow \emptyset}\right\}
$$

where $L_{Q \rightarrow \emptyset}=\frac{\operatorname{Pr}\left(p i v_{Q} \mid a\right)}{\operatorname{Pr}\left(p i v_{Q} \mid q\right)}$. Condition (7) simply states that a switch from $Q$ to $A$ (when using $L_{Q \rightarrow A}$ ) or from $Q$ to $\emptyset$ (when using $L_{Q \rightarrow \emptyset}$ ) is not desirable.

Note that if $L_{\emptyset \rightarrow A} \geq L_{Q \rightarrow A} \geq L_{Q \rightarrow \emptyset}$ we have that, for every type either (5) or (7) holds 
and abstention cannot be part of the equilibrium behavior of any type. Since $L_{\emptyset \rightarrow A} \geq L_{Q \rightarrow A}$ is equivalent to $L_{Q \rightarrow A} \geq L_{Q \rightarrow \emptyset}$ it follows that a necessary condition for abstention is that

$$
L_{\emptyset \rightarrow A}<L_{Q \rightarrow \emptyset}
$$

What does this condition means? Take for example a type such that $L_{\emptyset \rightarrow A}<\frac{\theta_{q}}{\theta_{a}}<L_{Q \rightarrow \emptyset}$ so we have that

$$
\begin{aligned}
& \theta_{a} \times \frac{\operatorname{Pr}\left(\text { piv }_{A} \mid a\right)}{2}-\theta_{q} \times \frac{\operatorname{Pr}\left(\text { piv }_{A} \mid q\right)}{2}<0 \\
& \theta_{q} \times \frac{\operatorname{Pr}\left(p i v_{Q} \mid q\right)}{2}-\theta_{a} \times \frac{\operatorname{Pr}\left(\text { piv }_{Q} \mid a\right)}{2}<0
\end{aligned}
$$

On one hand, $\theta_{a} \times \frac{\operatorname{Pr}\left(p i v_{A} \mid a\right)}{2}$ is the expected benefit for electing $A$ in state $a$ by voting for $A$ uninformatively while $\theta_{q} \times \frac{\operatorname{Pr}\left(p i v_{A} \mid q\right)}{2}$ is the expected utility loss from wrongly electing $A$ in state $q$ by voting for $A$ uninformatively. Therefore the left hand side of the first line in (9) is the expected net benefit of voting for $A$ without any information. Analogously, the left hand side of second line in (9) is the expected net benefit of uninformatively voting for $Q$. These two conditions imply that a voter with preferences $\frac{\theta_{q}}{\theta_{a}}$ that decides not to collect information maximizes her expected utility by not voting for $Q$ nor $A$. This rationally ignorant decision to abstain is the traditional swing voter's curse in $[11] .8$

It is immediate to see that if $p=\frac{1}{2}$ the set of types $\theta$ that changes her vote depending on the signal received has no mass: if a voter is willing to switch votes when the signals are uninformative she must be indifferent between the two candidates and by assumption indifference has measure 0 . Therefore, only uninformative strategies, such that the voter only supports one candidate or abstains, and informative strategies with information collection and voting behavior that depends on the signal need to be studied.

Recalling that a voting function is a pair $\left(v_{q}, v_{a}\right) \in\{Q, A, \emptyset\}^{2}$, there are 9 possible voting

\footnotetext{
${ }^{8}$ The condition $L_{\emptyset \rightarrow A}<L_{Q \rightarrow \emptyset}$ will end up being sufficient once we can show that there is always some type $\left(\theta_{q}^{\prime}, \theta_{a}^{\prime}\right)$ with sufficiently small $\theta_{q}^{\prime}$ and $\theta_{a}^{\prime}$ such that no information acquisition is optimal.
} 
functions. Since those strategies that involve information being used in the wrong way are not optimal for any positive mass of players we have the following:

Lemma 1 The voting strategies $A Q, A \emptyset$ or $\emptyset Q$ are not optimal for almost all types.

We are left with only six strategies that may occur in equilibrium with positive probability; three of these strategies involve no information acquisition and the remaining three strategies involve information acquisition. In principle voters can be separated into six different groups: $A$ and $Q$ strong supporters $\left(\mathcal{S S}^{A}\right.$ and $\mathcal{S S}^{Q}$ respectively), that vote in favor of their preferred candidate uninformatively; $A$ and $Q$ weak supporters $\left(\mathcal{W} \mathcal{S}^{A}\right.$ and $\mathcal{W S}^{Q}$ ), that support the candidate they ideologically lean to if the signal goes in favor of that candidate and abstain otherwise; abstainers $(\mathcal{A})$ who abstain uninformatively; and independents $(\mathcal{I})$ that collect information and follow the signal received. The question is which set of voters will emerge in equilibrium and which will not be part of the equilibrium.

To finish this section we will show that if a voter behaves as an independent the level of investment is bounded below and, if there is abstention in equilibrium, this bound is strictly above $\frac{1}{2}$. The next lemma states the result formally.

Lemma 2 A necessary condition for the independent behavior to be optimal with investment level $p$, is

$$
\left(\frac{p}{1-p}\right)^{2} \geq \frac{L_{Q \rightarrow \emptyset}}{L_{\emptyset \rightarrow A}}
$$

Moreover, if there is endogenous abstention with positive probability, i.e. $\frac{L_{Q \rightarrow \emptyset}}{L_{\emptyset \rightarrow A}}>1$, independents must invest a strictly positive amount.

Condition (10) follows by using (5) for $s_{a}$ and (7) for $s_{q}$ jointly. The second part is just an application of (8) which is the swing voter's curse. Let's discuss the intuition by focusing on a type with fairly balanced preferences, $\theta_{a} \sim \theta_{q}$, and low intensity; for example, $\theta_{a}$ and $\theta_{q}$ close to 0 . Since the swing voter's curse holds and the quality of information she collects is not enough to move her posteriors sufficiently in any direction (her intensity is low), she 
might prefer to abstain even after receiving information. If we also consider that by deciding not to collect any information she saves on information acquisition costs, she clearly prefers to abstain uninformatively.

Condition (10) implies that if in equilibrium the ratio of the probabilities of the relevant

events in the different states are such that $\max _{\theta \in[0,1]^{2}} \frac{P^{*}(\theta)}{1-P^{*}(\theta)}<\sqrt{\frac{L_{Q \rightarrow \emptyset}}{L_{\emptyset \rightarrow A}}}$ the set of independents would be empty. In such an equilibrium, moderate voters would be abstaining and collecting no information which contrasts with the fact that they are the ones that value information the most. The problem is that they value information the most because they need their posteriors to adjust the most but, given the quality of information they are willing to collect, those posteriors do not move enough.

Before proceeding to the characterization of equilibrium we need to study the information acquisition choice of each of the different groups of voters.

\subsection{Information acquisition}

Recall that there are three relevant investment functions: one for each group that collects information (independents and $A$ weak supporters and $Q$ weak supporters ). Let $P^{x}$ : $[0,1]^{2} \rightarrow\left[\frac{1}{2}, 1\right]$ be the information acquisition function of a voter type $\theta \in[0,1]^{2}$ planning on using one of the informative strategies $x \in\{Q A, \emptyset A, Q \emptyset\}$. Using (3) and Bayes' rule for each of the possible optimal strategies with investment and the information technology, we derive the optimal investment function implicitly as:

$$
\begin{aligned}
& C^{\prime}\left(P^{Q A}(\theta)\right)=\sum_{\omega \in\{q, a\}} \theta_{\omega} \frac{\operatorname{Pr}\left(\text { piv }_{Q} \cup \operatorname{piv}_{A} \mid \omega\right)}{2} \\
& C^{\prime}\left(P^{\emptyset A}(\theta)\right)=\sum_{\omega \in\{q, a\}} \theta_{\omega} \frac{\operatorname{Pr}\left(p i v_{A} \mid \omega\right)}{2} \\
& C^{\prime}\left(P^{Q \emptyset}(\theta)\right)=\sum_{\omega \in\{q, a\}} \theta_{\omega} \frac{\operatorname{Pr}\left(\text { piv }_{Q} \mid \omega\right)}{2}
\end{aligned}
$$

Let's focus on the first line of (11). The term $\frac{\operatorname{Pr}\left(p i v_{Q} \cup p i v_{A} \mid \omega\right)}{2}$ measures the expected impact 
on the chances of $A$ winning when a voter changes her vote from $Q$ to $A$. Because the signal is binary, the effect of information enters linearly on the expected utility (3). It follows that $\theta_{a} \frac{\operatorname{Pr}\left(p i v_{Q} \cup p i v_{A} \mid a\right)}{2}$ measures the gain from that marginal increment in A's chances of winning in the right state and $\theta_{q} \frac{\operatorname{Pr}\left(p i v_{Q} \cup p i v_{A} \mid q\right)}{2}$ measures the gain from the marginal reduction of $A^{\prime}$ 's chances of winning in the wrong state. Then, the right hand side of each line in (11) is the marginal benefit of increasing investment in quality of information conditional on a particular informative voting strategy. The first order condition for the choice of quality of information is just that the marginal benefit equates the marginal cost of information acquisition.

The first order condition (11) highlights the importance of our assumption regarding the two dimensional preferences: the amount of information acquisition depends on the actual levels of the utility losses. In contrast, the voting conditions (5) and (7) point out that it is the relative ratio of utility losses what matters for all voting decisions. ${ }^{9}$

Because we assume that the marginal cost of precision is infinitely large for a perfectly informative signal there is some upper bound strictly smaller than 1 on the maximum level of precision for all informative voting strategies. In particular, no informed voter will ever be perfectly informed. Comparing the first line with the second and third lines in (11) we can see that the information acquisition function is discontinuous. This discontinuity appears, for example, when a voter is indifferent between using an informative strategy that always calls to follow both signals and an informative strategy that involves abstention after one signal. This is directly related to the fact that the relevant pivotal events are different depending on which strategy is being used. In particular a strategy that calls to follow the signal always, making the voter switch from $A$ to $Q$ or viceversa, takes into account that there are more pivotal events that matter in comparison with a strategy that calls for abstention after some signal. Abstaining after some signal reduces the value of information.

\footnotetext{
${ }^{9}$ Our two dimensional preferences assumption is similar to assuming a particular private cost function as types. For example, let types be described now by $\tau_{a}=\frac{\theta_{a}}{\theta_{a}+\theta_{q}}$ and $\tau_{q}=1-\tau_{a}$, and let $c=\frac{1}{\theta_{a}+\theta_{q}}$. It follows that the first line of (11) turns into $c \times C^{\prime}\left(P^{Q A}(\theta)\right)=\sum_{\omega \in\{q, a\}} \tau_{\omega} \frac{\operatorname{Pr}\left(p i v_{Q} \cup p i v_{A} \mid \omega\right)}{2}$. Types are now described by an ideological parameter, $\tau_{a} \in[0,1]$, and a cost parameter $c \geq 1$. We thank the co-editor for pointing out this relation.
} 
Since the change in the value of information is discrete, voters with very similar preferences that decide to use different voting functions will collect very different qualities of information. For example, those that are planning on abstaining after some signal collect less information than independents despite having almost equal preferences.

\subsection{Existence and Characterization}

The information acquisition function is a function of the voter's type to the interval $\left[\frac{1}{2}, 1\right]$ so finding an equilibrium among all possible information acquisition functions requires the use of fixed point arguments in functional spaces. Usually a notion of compactness or monotonicity is needed to show the existence of a fixed point in those spaces. Unfortunately, in our case, neither compactness nor monotonicity are granted unless we severely restrict the information technology ${ }^{10}$. The key to the existence result is that we can separate the types that use each one of the strategies with cutoff functions that are smooth functions of the pivotal probabilities. At the same time, the information acquisition functions for almost every type change smoothly as functions of the probabilities of the relevant pivotal events. Since strategies are fully characterized by those pivotal probabilities finding a fixed point in the space of pivotal probabilities turns out to be equivalent to finding a fixed point in the space of strategies. This allows us to find an equilibrium by first describing it and then using its properties to actually show that there exists at least one.

The description of equilibrium reduces to finding which types use which strategy. The next Proposition summarizes the first result (characterization) that we discuss immediately after. Figure 1 is particularly useful to understand the next proposition

Proposition 1 Every equilibrium is characterized by

1. 6 different groups of voter types: strong supporters of each candidate $\left(\mathcal{S S}^{A}\right.$ and

\footnotetext{
${ }^{10}$ The quality of information is a discontinuous mapping of the preference parameters, even among voters who decide to collect information. The best response function is only a $C^{0}$ function almost everywhere. This precludes the application of fixed point arguments for infinite dimensional spaces (see [32], in particular, the equicontinutity requirement in Schauder's Fixed Point Theorem).
} 
$\left.\mathcal{S S}^{Q}\right)$, weak supporters of each candidate $\left(\mathcal{W S}^{A}\right.$ and $\left.\mathcal{W} \mathcal{S}^{Q}\right)$, abstainers $(\mathcal{A})$, and independents. (I); all groups of voter types have non empty interiors except for independents that might be empty.

2. The voting strategy $V^{*}(\theta)$ that verifies: $V^{*}(\theta)=A A$ for every $\theta \in \mathcal{S S}^{A}$ and $V^{*}(\theta)=$ $Q Q$ for every $\theta \in \mathcal{S S}^{Q} ; V^{*}(\theta)=\emptyset A$ for every $\theta \in \mathcal{W S}^{A}$ and $V^{*}(\theta)=Q \emptyset$ for every $\theta \in \mathcal{W S}^{Q} ; V^{*}(\theta)=\emptyset \emptyset$ for every $\theta \in \mathcal{A}$; and $V^{*}(\theta)=Q A$ for every $\theta \in \mathcal{I}$.

3. The information acquisition function $P^{*}(\theta)$ as defined in (11).

Although we cannot prove uniqueness of equilibrium, our partial characterization describes all symmetric Bayesian equilibria. The proof is organized in a sequence of steps. First, in order to determine the strategies played by each type we perform pairwise comparisons of strategies. We construct cutoffs functions that determine those types that are indifferent between two strategies. The description of the types that use each strategy is performed by partitioning the space of types using these cutoff functions. For example, when comparing the strategies $\emptyset A$ and $\emptyset \emptyset$ we define the function $g^{(\emptyset A)=(\emptyset \emptyset)}:[0,1] \rightarrow[0,1]$ such that the type $\left(\theta_{a}, g^{(\emptyset A)=(\emptyset \emptyset)}\left(\theta_{a}\right)\right)$ is indifferent between using the strategy $\emptyset$, collecting information optimally, and the strategy $\emptyset \emptyset$. Part of the proof is showing that this can be done in a sensible way and that it actually describes optimal behavior.

The proof continues by showing that there is abstention in equilibrium and it follows by contradiction. Essentially, by assuming that condition (8) does not hold we have that there is no endogenous abstention, i.e. $\mathcal{W S}^{A}, \mathcal{A}$, and $\mathcal{W} \mathcal{S}^{Q}$ are empty. It follows that the set $\mathcal{I}$ is not empty so the average voter is in fact informed. Since some voters are informed, conditional on the state $q(a)$, the event in which $Q$ is winning by one vote or tying is more (less) likely than the event in which $A$ is winning by one vote or tying; the swing voter's curse holds and condition (8) must hold in equilibrium. We then show that, in fact condition (8) is sufficient for endogenous abstention. That is done by showing that if condition (8) holds, then $\mathcal{W S}^{A}, \mathcal{A}$, and $\mathcal{W} \mathcal{S}^{Q}$ are not empty. 
Let's discuss some salient characteristics of the equilibrium. Strong supporters are sufficiently biased to overpower the effect of the maximum amount of information that they are willing to collect so they decide to remain uninformed. Independents and abstainers try to treat candidates similarly. The intensity of their preferences determines whether collecting information can help overcome any minor ideological bias they might have. Abstainers are rationally ignorant and do not vote for any candidate, and independents collect information to follow it.

The interesting groups are the $A$ weak supporters and $Q$ weak supporters . They are characterized by weakly biased preferences so they do not want to treat both candidates similarly if they are uninformed. This implies that behaving like abstainers is not optimal. Why are these types not behaving as independents? The maximum level of information they are willing to collect is not enough to overpower their ideological bias when the signal contradicts it, or acquiring information that overpowers their political bias is too costly and it is better to collect less information and do not use it that much. In essence these voters suffer the swing voter's curse because of the combination of ideology and information when the signal goes against their bias. So, in their case, abstention is actually driven by information.

Equilibria takes basically three forms that can be inferred from the description of each group of voters. A first type of equilibrium is presented in Figure 1. There we have a situation in which strong supporters locate on the "outside" and are surrounded only by weak supporters. Weak supporters are surrounded on the outside by strong supporters, but, on the inside, weak supporters with high intensity are surrounded by independents, and weak supporters with low intensity are surrounded by abstainers. Abstainers and independents have more balanced preferences, and they are aligned along the 45 degree line according to the intensity of preferences. Since it might be possible that behaving like independents is not optimal for some parameters, another form of equilibrium emerges when only abstainers are present in the 45 degree diagonal line. Figure 2 describes the third class of equilibria in 
which strong supporters share a boundary with independents. The main difference here is that weak supporters are moderately biased while some independents are characterized by a fairly strong bias but collect enough information to overpower it.

Figures 1 and 2 rely on certain symmetry assumptions: $\theta_{a}$ and $\theta_{q}$ are independent and symmetrically distributed around $\frac{1}{2}$, and $\xi_{A}=\xi_{Q}<\frac{1}{2}$. If we do not impose those symmetry conditions, we could have one side (say those biased towards $A$ ) where strong supporters are only surrounded by weak supporters, while the other side (biased towards $Q$ ) has strong supporters who are surrounded by both weak supporters and independents. When the electorate is asymmetric, i.e. one state is more likely than the other one or the preferences are skewed in favor of one particular candidate, the nice order presented in Figure 1 or 2 might be lost. The question is then, what can we say in general about the ideological order of voters and the voting strategies?

Recalling $g^{(\emptyset A)=(\emptyset \emptyset)}:[0,1] \rightarrow[0,1]$, the cutoff function such that all types $\left(\theta_{a}, g^{(\emptyset A)=(\emptyset \emptyset)}\left(\theta_{a}\right)\right)$ are indifferent between the strategies $\emptyset A$ and $\emptyset \emptyset$, we define $g^{(Q \emptyset)=(\emptyset \emptyset)}:[0,1] \rightarrow[0,1]$ as the cutoff function for the strategies $Q \emptyset$ and $\emptyset \emptyset$. In the Appendix we show that if $\theta_{q}<g^{(\emptyset A)=(\emptyset \emptyset)}\left(\theta_{a}\right)$ the type $\left(\theta_{a}, \theta_{q}\right)$ prefers the strategy $\emptyset \emptyset$ to the strategy $\emptyset A$ and if $\theta_{q}<g^{(Q \emptyset)=(\emptyset \emptyset)}\left(\theta_{a}\right)$ the type $\left(\theta_{a}, \theta_{q}\right)$ prefers the strategy $\emptyset \emptyset$ to the strategy $Q \emptyset$. With these function in mind, and considering that independents collect a strictly positive amount of information (see Lemma 2), for low values of $\theta_{a}$ and $\theta_{q}$ we have that abstainers exist because $g^{(\emptyset A)=(\emptyset \emptyset)}\left(\theta_{a}\right)<\theta_{q}<g^{(Q \emptyset)=(\emptyset \emptyset)}\left(\theta_{a}\right)$ for some $\theta$. For sufficiently low intensity, the separation of types close to the origin is given by the cutoff function between $Q$ strong supporters and $Q$ weak supporters, the cutoff function between $Q$ weak supporters and abstainers, and the respective cutoff functions for $A$ supporters. Then

Proposition 2 In any equilibrium there is some $\vartheta>0$ such that for every type that verifies $\theta_{a}+\theta_{q} \leq \vartheta$ the group of voters are ordered clockwise in the following way: $\mathcal{S S}^{A}, \mathcal{W} \mathcal{S}^{A}, \mathcal{A}$, $\mathcal{W} \mathcal{S}^{Q}$ and $\mathcal{S S}^{Q}$

The fact that voters are ordered in this way implies that the quality of information is 


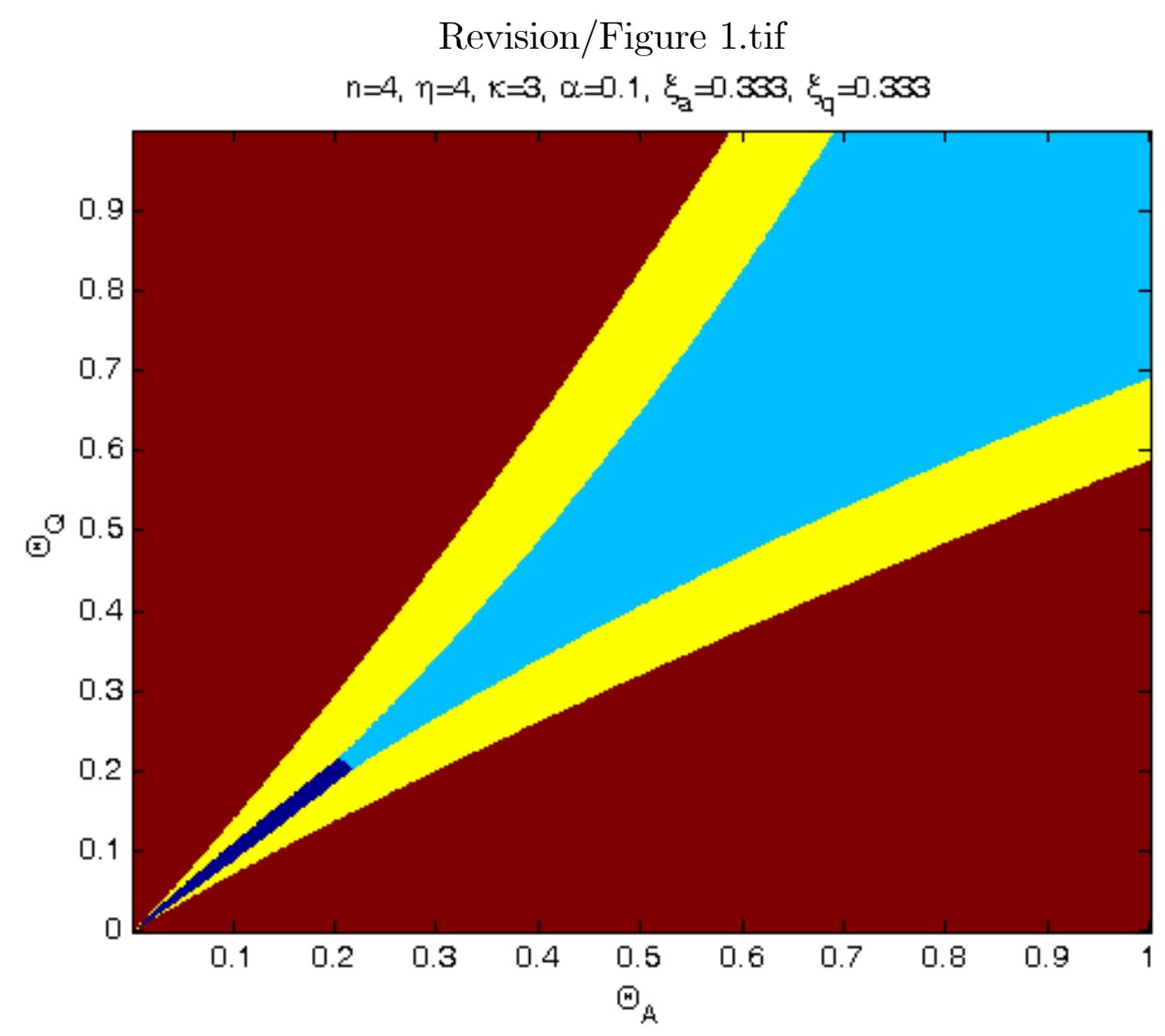

Figure 1: Strong supporters are in red, weak supporters in yellow, independents in light blue and abstainers in dark blue. The losses $\theta_{a}$ and $\theta_{q}$ are beta random variables with parameters $(2,2)$ and the committee consists of $4(n=4)$ members that are partisan with $10 \%$ probability $(\alpha=0.1)$ and are evenly split between the voting options $\left(\xi_{a}=\xi_{q}=\xi_{\varnothing}=\frac{1}{3}\right)$. The cost function is $C(p)=4\left(p-\frac{1}{2}\right)^{3}$.

not monotonic in ideology for low intensity. First of all, let's concentrate on types with the same level of intensity; say $\theta_{a}+\theta_{q}=\beta$ and assume that $\beta$ is sufficiently low so types are ordered like in Proposition 2. Consider the case in Figure 1 starting from $\theta_{a}=0$ and $\theta_{q}=\beta$ and increasing $\theta_{a}$ down the "isointensity" line $\theta_{a}+\theta_{q}=\beta$. Information is nil when the voter is a $Q$ strong supporter, grows when the voter is a $Q$ weak supporter, and is nil again when the voter becomes abstainer; then information is positive when the voter becomes a $A$ weak supporter to be nil again when the voter is a $A$ strong supporter. Note that centrist voters are those that value the information the most but, when their intensity is low, their posteriors do not change much after any signal and saving on information acquisition is optimal, so they decide to remain uninformed and abstain. On the other hand if $\beta$ is sufficiently large we could move from $Q$ weak supporters to independents and then to $A$ weak supporters. 


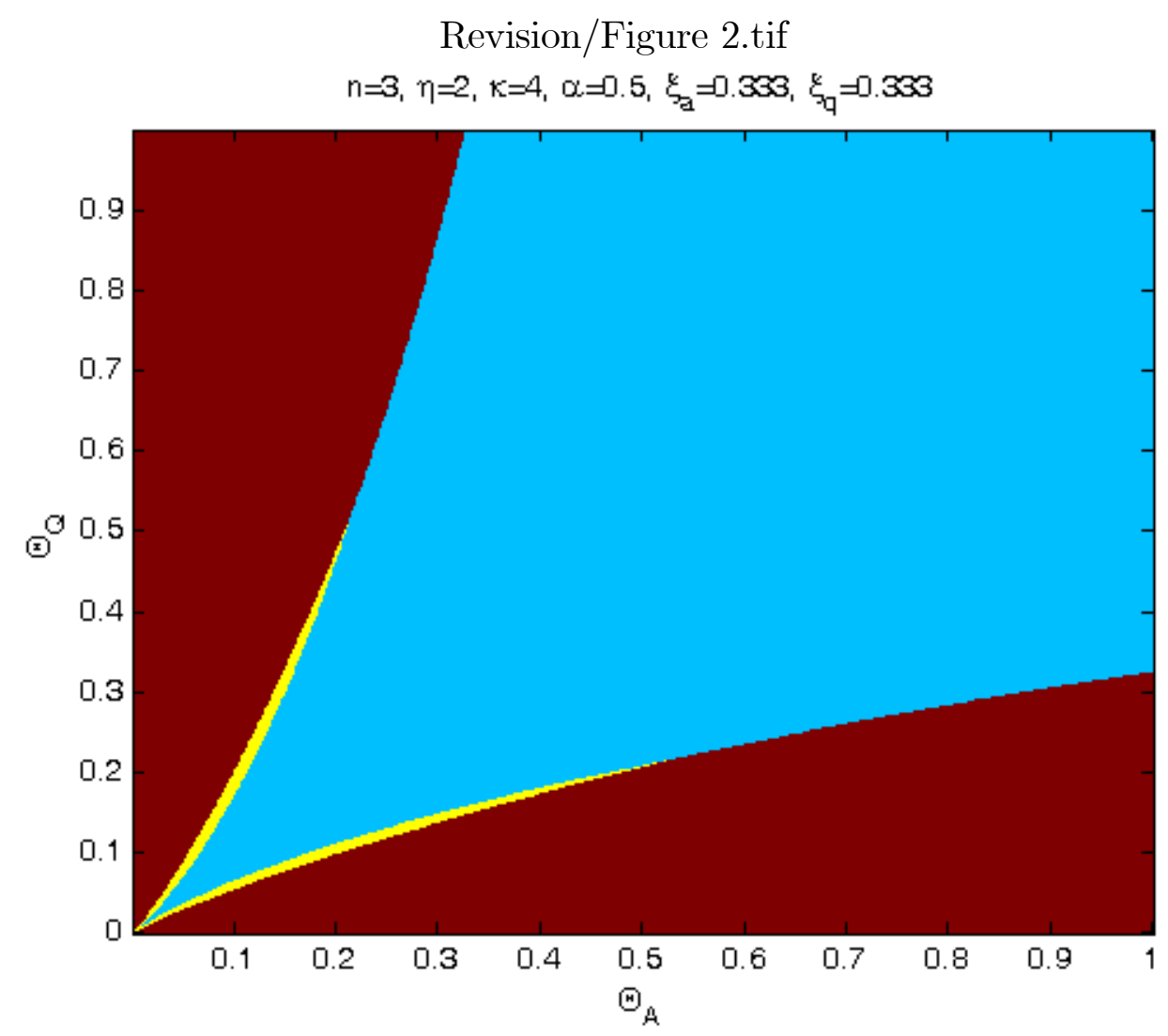

Figure 2: Strong supporters are in red, weak supporters in yellow, independents in light blue and abstainers in dark blue. The losses $\theta_{a}$ and $\theta_{q}$ are beta random variables with parameters $(1,2)$ and the committee consists of $3(n=3)$ members that are partisan with $10 \%$ probability $(\alpha=0.1)$ and are evenly split between the voting options $\left(\xi_{a}=\xi_{q}=\xi_{\varnothing}=\frac{1}{3}\right)$. The cost function is $C(p)=2\left(p-\frac{1}{2}\right)^{4}$. The size of abstainers is significantly small.

We can argue that more centrist voters will collect more information. In this case, centrist voters scape the swing voter's curse by collecting high quality information that shifts their posteriors enough.

We are ready now to prove existence. The arguments are in line with the discussion at the beginning of the section: a best response in our voting game is fully captured by the pivotal probabilities, so finding a fixed point in the space of pivotal probabilities is finding an equilibrium in the voting game.

Proposition 3 There exists a symmetric Bayesian equilibrium. 


\section{Applications}

\subsection{Some comparative statics}

Information and abstention in the limit The "parameters" of the cutoff functions $g$ are endogenous variables so we have only provided a partial characterization of equilibrium. Comparative statics analysis turns out to be significantly difficult since we require a complete and full characterization of equilibrium. In this section we take a short cut and discuss properties of the equilibrium when the number of voters is large. In particular we focus on the following questions, what is the ideology of the voters that collect information, and what is the ideology of the voters that abstain? Unlike [11] and [12], in our model no nonpartisan voter abstains when the electorate becomes large. The intuition hinges on the fact that investment in information is small in the limit so the effect of the swing voter's curse disappears. The smaller the quantity of information collected by the average player, the more a player relies on her own private ideological bias so the less willing she is to abstain. As a consequence weak supporters and abstainers disappear when $n$ is large.

Proposition 4 When the electorate is sufficiently large, $P(\theta) \rightarrow \frac{1}{2}$ for all $\theta \in[0,1]$ and the probability of a non partisan voter abstaining goes to 0 .

Contrasts this, for example, with [27] where, even though there is a large number of voters, some positive mass of voters use the information and some positive mass of voters abstain. His result relies on the fact that voters exogenously receive information and those that are better informed want to vote while those that are less informed prefer to abstain. Our paper points out that, those results (positive abstention and positive amount of information used in equilibrium by the average voter) are directly related to the assumption about exogenous information in costless models of voting.

Changes in partisan support Elections of House Representatives, Senators and Governors are usually a particular state affair. Each state might have a different distribution of 
registered republicans and democrats (partisan voters). The level of abstention and the level of information of the average voter also differs from state to state. How does the distribution of partisan support affect the voters' willingness to collect information and abstain? In particular, we now focus on identifying how the set of voters using different strategies changes when the number of voters is large. This exercise links the presence of partisan voters with the response of non- partisan voters in electorates that are sufficiently large.

Lemma 3 When the electorate is sufficiently large the set of $X$ strong supporters verifies

$$
\int_{\theta \in \mathcal{S S}^{X}} f(\theta) d \theta \approx \frac{1}{2}+\alpha \frac{\left(\xi_{-X}-\xi_{X}\right)}{2}
$$

For a sufficiently large number of voters the proportion of votes in favor of one or the other candidate is close to being evenly split. Moreover, (12) shows that strong supporters compensate for the bias introduced by partisan voters. For example, when $\xi_{A}$ increases (while $\xi_{Q}$ is constant, which means less partisan abstention), the set of $A$ strong supporters decreases. In terms of Figures 1 and 2, increments in $\xi_{A}$ induce a clockwise shift. This implies that for a sufficiently large number of voters, the higher the partisan vote in favor of $A$, the more ideologically biased toward that candidate are the voters who collect information.

Note that an increment on the proportion of non partisan voters $(\alpha)$ has a differential effect depending on the proportion of each candidate non partisan supporters. Assume the case $\xi_{A}>\xi_{Q}$. Then increasing $\alpha$, increases the probability of a vote for $A$ by a partisan voter more than what it increases the probability of a vote for $Q$ by a partisan voter. As we had before, the set of $Q$ strong supporters grows, while the set of $A$ strong supporters shrinks; and, again we have a clockwise shift in Figures 1 and 2 so voters that are leaning towards $A$ are the ones that are more likely to collect information. The opposite happens if $\xi_{A}<\xi_{Q}$.

To sum up, a change in the electorate that raises the proportion of partisan voters who support candidate $A$ with respect to the proportion of partisan voters that support candidate $Q$, implies that the non partisan voters who are closer ideologically to candidate $A$ will be 
the voters that actually collect information in equilibrium. And this, in turns, implies that voters that are closer to candidate $A$ ideologically are also those who might decide to abstain. Consequently, the ideology of independent voters and abstainers is directly related to the relative proportions of partisan voters and who collects information depends on which candidate has more partisan support. A testable implication of this paper is that the more partisan support for one candidate, the more biased towards that candidate are those that collect information and potentially abstain. To our knowledge we are the first ones to uncover this relation.

\subsection{The role of flexible preferences}

In the model presented here, preferences are described by two parameters. It is traditional in voting models to assume that utility losses are perfectly and inversely correlated $\left(\theta_{q}^{i}+\theta_{a}^{i}=\delta_{1}\right.$ for all $i) .{ }^{11}$ This assumption is sufficient to describe the voting strategy after information has been collected, as can be seen in expressions (5) and (7). On the other hand, the expressions in (11) show that the absolute levels of these losses are relevant in terms of information acquisition. We now illustrate why allowing for flexible preferences matters theoretically when information is endogenous. Essentially, restricting preferences diminishes the model's capacity of properly capturing abstention as a social phenomenon.

Assume now that preferences are described by restricting $\theta_{q}^{i}+\theta_{a}^{i}=1$ for all $i$ and that $\widehat{F}$ is the prior distribution of types $\theta_{a}^{i} \in[0,1]$; a slight modification of Propositions 1 and 3 allows us to use the same results obtained in the previous sections to study this restricted model using now cutoff types instead of cutoff functions. ${ }^{12}$ Let $\underline{p}$ be defined as the lower

\footnotetext{
${ }^{11}$ Assumptions presenting heterogeneity as $\theta_{q}^{i}-\theta_{a}^{i}=\phi^{i} \in[-1,1]$ or $\frac{\theta_{q}^{i}}{\theta_{a}^{i}}=\phi^{i} \in[0, \infty]$ suffer the same drawback presented here.

${ }^{12}$ Proposition (3) uses the hyperdifuse assumption to assure that no mass of types is concentrated on any of the cutoffs functions. This allows for a simple integration over types to capture the probability of a giving vote and makes each set of voters "smooth" with respect to changes in the "pivotal" probabilities. When we reduce the dimension of the space of types, assuming that $\widehat{F}$ has no mass points is sufficient to assure that cutoff types have no mass. The key to this extension is realizing that existence follows by a fixed point in the space of pivotal probabilities and not in the space of best responses per se.
} 
investment possible by independents in Lemma 2: $\frac{\underline{p}}{1-\underline{p}}=\sqrt{\frac{L_{Q \rightarrow \emptyset}}{L_{\emptyset \rightarrow A}}}$.

Under symmetric conditions ${ }^{13}$ we have that every informed independent actually collects the same amount of information. This happens because under symmetry the equilibrium probability of $A$ winning (losing) by one vote in state $a$ is the same as the equilibrium probability of $Q$ winning (losing) by one vote in state $q$, and the equilibrium probability of a tie is the same in both states; it follows that $\frac{\operatorname{Pr}\left(p i v_{Q} \cup p i v_{A} \mid a\right)}{2}=\frac{\operatorname{Pr}\left(p i v_{Q} \cup p i v_{A} \mid q\right)}{2}=\gamma$ and (11) yields $C^{\prime}\left(P^{Q A}(\theta)\right)=\gamma \cdot{ }^{14}$ If, for a given electorate size, we have that $C^{\prime}(\underline{p}) \leq \gamma$ then $\underline{p} \leq$ $P^{Q A}(\theta)$ and all voters that have fairly balanced preferences will decide to collect information $P^{Q A}(\theta)$ and follow the signal. In this case no abstainers emerge in equilibrium. We could have concluded that abstention by uninformed voters is not an equilibrium phenomenon. ${ }^{15}$ If, on the other hand for a given electorate $C^{\prime}(\underline{p})>\gamma$ we must have that all moderate voters do not want to collect information and independents are not present in equilibrium. Because only weak supporters are actually collecting information, we could have concluded that only "intermediate levels" of ideology collect information as in [22].

\subsection{The correlation between information and abstention}

It is well documented that education affects turnout (see [29] and references there) but there is more disagreement regarding the mechanism by which they are related. [14] finds that education is correlated with political knowledge while [20] finds that education provides the information processing ability to collect and understand political information. In turns, this ability is the one that creates the relation between education and abstention (see also [3] for a similar argument). Note that these arguments rely on the fact that education is related to information, and information is then related to the desire to vote. ${ }^{16}$ In this

\footnotetext{
${ }^{13}$ Essentially that $F\left(\theta_{a}\right)$ is symmetric around $\frac{1}{2}$ and $\xi_{A}=\xi_{Q}<\frac{1}{2}$.

${ }^{14}$ Details can be provided upon request.

${ }^{15}$ Moreover, if the equilibrium pivotal probabilities that emerge in Figure 2 are also equilibrium pivotal probabilities of the game with restricted preferences no voter in the counterdiagonal abstains. In this case, we would have concluded that abstention is not an equilibrium phenomenon!!!

${ }^{16}$ For an argument that does not link education and turnout via information see [29] who argue that educated people tend to register more and this is what drives the relation between education and turnout.
} 
subsection we illustrate how our model contributes to this debate. Essentially, the particular distribution of voters' ideology affects the aggregate correlation between information and abstention and, hence, also affects the estimation of the effect of education on abstention. To our knowledge, no empirical work has incorporated the particular ideology in capturing the effect of education on turnout and, hence, has potentially underestimated the effect of education. $^{17}$

Let $\operatorname{Pr}(v \neq \emptyset \mid P, \omega)$ be the probability of voting conditional on the quality of the information $P$ and on the state $\omega$. It is obvious that $\frac{d \operatorname{Pr}(v \neq \emptyset \mid P, \omega)}{d P}=0$ for all those who strictly prefer to be uninformed ( $A$ strong supporters, $Q$ strong supporters and abstainers) as well as for those who behave as independents. On the other hand, in state $a$ turnout of $A$ weak supporters is positively correlated with information while turnout of $Q$ weak supporters is negatively correlated with information. In state $q$, of course, the correlation between information and turnout goes in the opposite direction for weak supporters. This implies that, at the aggregate level, the correlation between information and turnout depends on the relative size of the sets of $A$ weak supporters and $Q$ weak supporters.

\section{Conclusions}

In this paper we present a voting model with abstention and endogenous information acquisition using two interdependent innovations: first, committee members' preferences differ on the levels of both ideology and intensity, and, second, voters select the precision of the signal they receive. The combination of these two features leads to voters collecting information of different qualities in equilibrium. Understanding the role of information on voting requires characterizing who collects information, how much information is collected, and how this information is used. While variation in the ideological dimension is enough to show that rel-

\footnotetext{
[20] also recognizes this effect.

${ }^{17}$ Although focusing on the impact of media bias, [9], find that the availability of independent TV channels decreases turnout in Russia. They do control for initial ideology in calculating the effect of vote choice. They attribute the turnout effect to negative campaigning.
} 
atively centrist voters acquire information, ${ }^{18}$ differences in intensity give raise to interesting effects on the level of information that is acquired and on how this information affects voting behavior.

We show that information acquisition is not monotonic in ideology or intensity. Moreover, the optimal information acquisition function is discontinuous even among voters who collect some information. This suggests that the link between fundamental characteristics of voters and information acquisition is more intricate than previously thought. For example, if we associate education with higher levels of concern (or its analogous, lower costs for information acquisition), a voter with relatively pronounced ideological preferences might collect significantly less information than another voter with the same educational level but more moderate ideological preferences.

We also show that in order to find a clear pattern between information and abstention we need to focus the analysis on voters with similar ideological bias: some voters with a mild bias in favor of one candidate are more likely to vote the more informed they are, while other voters with a mild bias in favor of the other candidate are more likely to abstain the more informed they are. In our more general model, some voters abstain even if they have much at stake in the election and have relatively strong evidence in favor of one candidate. Abstention is not simply the result of poor information, but of a more complex interaction between preferences and information.

We provide a novel test of the relation between information and abstention. In particular or model predicts a stark contrast on the way information affects turnout between opposing ideologies. For example, those leaning towards the winner that decided to abstain despite having collected information should be on average less informed than those leaning towards the loser that decided to abstain. We also provide a novel prediction about the correlation between partisan support and the ideology of those that collect information. In our model the ideology of informed voters is positively correlated with the relative weights of partisan

\footnotetext{
${ }^{18}$ See [25] and [21] for two models with restricted preferences that generate different predictions about who collects information.
} 
support: the more right leaning an electorate is the more right leaning the ideology of informed voters.

We finish by discussing a particular application of the model. For example, in a presidential race candidates need to decide which topics to discuss during the campaign, considering how much effort the voters are willing to exert in understanding the message. If there is high variance on the preferences of non partisan voters, a relatively polarized electorate, a political campaign that provides detailed information might not be optimal. This is because getting the message requires a level of effort from the voters that, on average, they are not willing to exert. On the other hand, simple slogans with half but fast truths might be more effective. Note also that this strategy will affect turnout but will not swing voters since it targets weak supporters. In contrast, when the electorate is less polarized, voters are more willing to process information and issues can be discussed in more depth. This strategy mostly swing voters that are deeply concern with the outcome of the election since it targets independents. A fully developed model of the political campaign game between two candidates is left for future research.

\section{References}

[1] Austen-Smith, D., Banks, J. S., 1996. Information aggregation, rationality, and the condorcet jury theorem. The American Political Science Review 90 (1), 34-45.

[2] Battaglini, M., Morton, R., Palfrey, T., 2010. The swing voter's curse in the laboratory. Review of Economic Studies 77 (1), 61-89.

[3] Brady, H., Verba, S., Schlozman, K., 1995. Beyond SES: A resource allocation model of political participation. American Political Science Review 89 (2), 271-294.

[4] Cai, H., 2009. Costly participation and heterogeneous preferences in informational committees. Rand Journal of Economics 40 (1), 173-189.

[5] Caplin, A., Nalebuff, B., January 1991. Aggregation and social choice: A mean voter theorem. Econometrica 59 (1), 1-23.

[6] Coupé, T., Noury, A. G., 2004. Choosing not to choose: On the link between information and abstention. Economics Letters 84 (2), 261-265. 
[7] Davis, O., Hinich, M., Ordeshook, P., 1970. An expository development of a mathematical model of the electoral process. American Political Science Review 64 (2), 426-448.

[8] DeGroot, M. H., 2004. Optimal Statistical Decisions, 2nd Edition. John Wiley Sons, New Jersey.

[9] Enikolopov, R., Petrova, M., Zhuravskaya, E., December 2011. Media and political persuasion: Evidence from russia. American Economic Review 101 (7), 3253-3285.

[10] Feddersen, T., 2004. Rational choice theory and the paradox of not voting. The Journal of Economic Perspectives 18 (1), 99-112.

[11] Feddersen, T., Pesendorfer, W., 1996. The swing voter's curse. The American Economic Review 86 (3), 408-24.

[12] Feddersen, T., Pesendorfer, W., 1999. Abstention in elections with asymmetric information and diverse preferences. The American Political Science Review 93 (2), 381-98.

[13] Feddersen, T., Sandroni, A., 2006. Ethical voters and costly information acquisition. Quarterly Journal of Political Science 1 (3), 187-311.

[14] Galston, W. A., 2001. Political knowledge, political engagement, and civic education. Annual Review of Political Science 4, 217-34.

[15] Gentzkow, M., 2006. Television and voter turnout. The Quarterly Journal of Economics $121(3), 931-972$.

[16] Gerardi, D., Yariv, L., 2008. Information acquisition in committees. Games and Economic Behavior 62 (2), 436-459.

[17] Gerling, K., Kiel, A., Schulte, E., Grüner, H. P., 2003. Information acquisition and decision making in committees: A survey. ECB Working Paper No. 256.

[18] Gershkov, A., Szentes, B., 2009. Optimal voting schemes with costly information acquisition. Journal of Economic Theory 144 (1), 36-68.

[19] Ghirardato, P., Katz, J. N., 2006. Indecision theory: Quality of information and voting behavior. Journal of Public Economic Theory 8 (3), 379-399.

[20] Jackson, R. A., July 1995. Clarifying the relationship between education and turnout. American Politics Research 23 (3), 279-299.

[21] Larcinese, V., 2007. Does political knowledge increase turnout? evidence from the 1997 british general election,. Public Choice 131, 387-411.

[22] Larcinese, V., 2009. Information acquisition, ideology and turnout: Theory and evidence from britain. Journal of Theoretical Politics 21 (2), 237-276.

[23] Lassen, D. D., 2005. The effect of information on voter turnout: Evidence from a natural experiment. American Journal of Political Science 49 (1), 103-118. 
[24] Li, H., 2001. A theory of conservatism. The Journal of Political Economy 109 (3), 617636.

[25] Martinelli, C., 2006. Would rational voters acquire costly information? Journal of Economic Theory 129 (1), 225-251.

[26] Martinelli, C., 2007. Rational ignorance and voting behavior. International Journal of Game Theory 35 (3), 315-394.

[27] McMurray, J. C., 2010. Empirical evidence of strategic voter abstention, Working Paper.

[28] McMurray, J. C., 2011. Aggregating information by voting: The wisdom of the experts versus the wisdom of the masses. Review of Economic Studies(forthcoming).

[29] Milligan, K., Moretti, E., Oreopoulos, P., 2004. Does education improve citizenship? evidence from the united states and the united kingdom. Journal of Public Economics 88 (9-10), 1667-1695.

[30] Palfrey, T. R., Poole, K. T., 1987. The relationship between information, ideology, and voting behavior,. American Journal of Political Science 31 (3), 511-530.

[31] Persico, N., 2004. Committee design with endogenous information. The Review of Economic Studies 71 (1), 165-91.

[32] Rudin, W., 1973. Functional Analysis, 2nd Edition. MacGraw- Hill Book Company.

[33] Shotts, K. W., 2006. A signaling model of repeated elections. Social Choice and Welfare $27,251-261$.

[34] Wattenberg, M. P., McAllister, I., Salvanto, A., 2000. How voting is like taking a SAT test: An analysis of american voter rolloff. American Politics Research 28 (2), 234-250. 


\section{A Appendix}

Proof of Lemma 1 We prove the case $A \emptyset$; the cases $\emptyset Q$ and $A Q$ are analogous. If a non partisan voter uses $A \emptyset,(5)$ and (7) give that

$$
\begin{aligned}
\frac{\operatorname{Pr}\left(q \mid s_{q}, p\right)}{\operatorname{Pr}\left(a \mid s_{q}, p\right)} & \leq \frac{\theta_{a}}{\theta_{q}} \min \left\{L_{Q \rightarrow A}, L_{\emptyset \rightarrow A}\right\} \\
& \leq \frac{\theta_{a}}{\theta_{q}} \max \left\{L_{Q \rightarrow A}, L_{Q \rightarrow \emptyset}\right\} \leq \frac{\operatorname{Pr}\left(q \mid s_{a}, p\right)}{\operatorname{Pr}\left(a \mid s_{a}, p\right)}
\end{aligned}
$$

which is a contradiction since $p>\frac{1}{2}$. If $p=\frac{1}{2}$, it is optimal only for types that satisfy $\frac{\theta_{q}}{\theta_{a}}=\min \left\{L_{Q \rightarrow A}, L_{\emptyset \rightarrow A}\right\}=\max \left\{L_{Q \rightarrow A}, L_{Q \rightarrow \emptyset}\right\}$.

Proof of Lemma 2 If $L_{Q \rightarrow \emptyset}>L_{\emptyset \rightarrow A}$, a voter that is willing to follow the signal after every realization must verify, condition (5) for $s_{a}$ and (7) for $s_{q}$. Hence it must be that $\frac{\theta_{q}}{\theta_{a}} \frac{1-p}{p} \leq L_{\emptyset \rightarrow A}$ and $\frac{\theta_{q}}{\theta_{a}} \frac{p}{1-p} \geq L_{Q \rightarrow \emptyset}$, and together give the condition (10). Recalling that, as discussed in the main text, a necessary condition for abstention is

$$
L_{Q \rightarrow \emptyset}>L_{Q \rightarrow A}>L_{\emptyset \rightarrow A}
$$

so it follows that $(10)$ implies $\left(\frac{p}{1-p}\right)^{2}>1$ and, $p>\frac{1}{2}$ is necessary.

\section{Proof of Proposition 1}

Cutoff functions In order to describe the equilibrium we need to define the cutoff functions discussed in the text.

Let $\Delta \mathcal{U}^{i}\left(\left(v_{q} v_{a}\right),\left(v_{q}^{\prime} v_{a}^{\prime}\right) \mid \theta_{q}, \theta_{a}\right)$ be the difference between the utility derived from using $v_{q} v_{a}$ and the utility from using the strategy $v_{q}^{\prime} v_{a}^{\prime}$ and acquiring information optimally for each strategy which is:

$$
\mathcal{U}^{i}\left(P^{v_{q} v_{a}}(\theta), v_{q} v_{a} \mid \theta\right)-C\left(P^{v_{q} v_{a}}(\theta)\right)-\left(\mathcal{U}^{i}\left(P^{v_{q}^{\prime} v_{a}^{\prime}}(\theta), v_{q}^{\prime} v_{a}^{\prime} \mid \theta\right)-C\left(P^{v_{q}^{\prime} v_{a}^{\prime}}(\theta)\right)\right)
$$

We define the function $g^{\left(v_{q} v_{a}\right)=\left(v_{q}^{\prime} v_{a}^{\prime}\right)}\left(\theta_{a}\right)$ such that the type $\theta=\left(g^{\left(v_{q} v_{a}\right)=\left(v_{q}^{\prime} v_{a}^{\prime}\right)}\left(\theta_{a}\right), \theta_{a}\right)$ is indifferent between using the strategy $\left(v_{q} v_{a}\right)$ and the strategy $\left(v_{q}^{\prime} v_{a}^{\prime}\right)$; implicitly we define the cutoff functions by the condition $\Delta \mathcal{U}^{i}\left(\left(v_{q} v_{a}\right),\left(v_{q}^{\prime} v_{a}^{\prime}\right) \mid g^{\left(v_{q} v_{a}\right)=\left(v_{q}^{\prime} v_{a}^{\prime}\right)}\left(\theta_{a}\right), \theta_{a}\right)=0$. In some cases we cannot assure that $g$ exists so we need to work with a function that maps mistakes in state $q$ to mistakes in state $a$. In such cases we are going to use the letter $h$ and define it implicitly as $\Delta \mathcal{U}^{i}\left(\left(v_{q} v_{a}\right),\left(v_{q}^{\prime} v_{a}^{\prime}\right) \mid \theta_{q}, h^{\left(v_{q} v_{a}\right)=\left(v_{q}^{\prime} v_{a}^{\prime}\right)}\left(\theta_{q}\right)\right)=0$.

Although $g$ and $h$ are functions of every voter's strategy, the effect of every voter except $i$ is aggregated via the pivotal probabilities. Both arguments are omitted in order to simplify notation. Second, these functions are defined beyond $[0,1]^{2}$. Third, for the cutoff function between independents and abstainers, we cannot show that $g^{(Q A)=(\emptyset \emptyset)}\left(\theta_{a}\right)$ or $h^{(Q A)=(\emptyset \emptyset)}\left(\theta_{q}\right)$ 
always exists. Nevertheless, we can show that, at least one of them exists. Moreover, for every pair $h$ and $g$ that is well defined, we can show that $h=g^{-1} \cdot{ }^{19}$ Finally, contrary to all other cases, it may be that $g^{(Q A)=(\emptyset \emptyset)}\left(\theta_{a}\right)>1\left(\right.$ or $\left.h^{(Q A)=(\emptyset \emptyset)}\left(\theta_{q}\right)>1\right)$ for all $\theta \in[0,1]^{2}$. In that case, being an abstainer is always better than being independent.

The next Remarks are useful to define each one of the sets of voters. The proofs are provided immediately below the statements.

Remark $1 g^{(A A)=(\emptyset A)}\left(\theta_{a}\right)<g^{(\emptyset A)=(\emptyset \emptyset)}\left(\theta_{a}\right)$ for every $\theta_{a}>0$

Let $\mathcal{L}(x)=C^{\prime}(x) x-C(x)$ and using (3), (11) and (14) we have that $g^{(\emptyset A)=(\emptyset \emptyset)}\left(\theta_{a}\right)$ and $g^{(A A)=(\emptyset A)}\left(\theta_{a}\right)$ are respectively defined as

$$
\begin{gathered}
\frac{\mathcal{L}\left(P^{(\emptyset A)}\left(g^{(\emptyset A)=(\emptyset \emptyset)}\left(\theta_{a}\right), \theta_{a}\right)\right)}{g^{(\emptyset A)=(\emptyset \emptyset)}\left(\theta_{a}\right)} \equiv \frac{\operatorname{Pr}\left(p i v_{A} \mid q\right)}{2} \\
\frac{\mathcal{L}\left(P^{(\emptyset A)}\left(g^{(A A)=(\emptyset A)}\left(\theta_{a}\right), \theta_{a}\right)\right)}{\theta_{a}} \equiv \frac{\operatorname{Pr}\left(p i v_{A} \mid a\right)}{2}
\end{gathered}
$$

Applying the implicit function theorem to (15) and (16) it follows that $g^{(\emptyset A)=(\emptyset \emptyset)}\left(\theta_{a}\right)$ is increasing and strictly convex and $g^{(\emptyset A)=(A A)}\left(\theta_{a}\right)$ is increasing and strictly concave. Using properties of convex and concave functions we have that $\frac{g^{(\emptyset A)=(\emptyset \emptyset)}\left(\theta_{a}\right)}{\theta_{a}}>L_{\emptyset \rightarrow A}>\frac{g^{(A A)=(\emptyset A)}\left(\theta_{a}\right)}{\theta_{a}}$.

Remark $2 g^{(Q \emptyset)=(\emptyset \emptyset)}\left(\theta_{a}\right)<g^{(Q \emptyset)=(Q Q)}\left(\theta_{a}\right)$ for every $\theta_{a}>0$

It follows the same line used in Remark 1.

Remark $3 g^{(\emptyset A)=(Q A)}\left(\theta_{a}\right)<g^{(Q \emptyset)=(\emptyset \emptyset)}\left(\theta_{a}\right)$ for every $\theta_{a}>0$ where $g^{(\emptyset A)=(Q A)}\left(\theta_{a}\right)$ verifies $\left(h^{(\emptyset A)=(Q A)}\right)^{-1}=g^{(\emptyset A)=(Q A)}$

Let the pair $\left(\widetilde{\theta}_{q}, \widetilde{\theta}_{a}\right)$ be such that $\widetilde{\theta}_{q}=g^{(Q \emptyset)=(\emptyset \emptyset)}\left(\widetilde{\theta}_{a}\right)$ so $(3),(11),(14)$ and the definition of $\mathcal{L}(x)$ give that

$$
\frac{\mathcal{L}\left(P^{Q \emptyset}\left(\widetilde{\theta}_{q}, \widetilde{\theta}_{a}\right)\right)}{\widetilde{\theta}_{a}}=\frac{\operatorname{Pr}\left(\operatorname{piv}_{Q} \mid a\right)}{2}
$$

Using again $(3),(11)$, and (14) we have that the type $\left(\widetilde{\theta}_{q}, \widetilde{\theta}_{a}\right)$ prefers $(Q A)$ to $(\emptyset A)$ if

$$
\frac{\mathcal{L}\left(P^{Q A}\left(\widetilde{\theta}_{q}, \widetilde{\theta}_{a}\right)\right)-\mathcal{L}\left(P^{\emptyset A}\left(\widetilde{\theta}_{q}, \tilde{\theta}_{a}\right)\right)}{\widetilde{\theta}_{a}} \geq \frac{\operatorname{Pr}\left(p i v_{Q} \mid a\right)}{2}
$$

Using the second line of (11) we have that the condition (18) is equivalent to

$$
\begin{aligned}
& \widetilde{\theta}_{a} \frac{\operatorname{Pr}\left(\operatorname{piv}_{Q} \mid a\right)}{2}+C\left(P^{Q A}\left(\widetilde{\theta}_{q}, \widetilde{\theta}_{a}\right)\right)-C\left(P^{\emptyset A}\left(\widetilde{\theta}_{q}, \widetilde{\theta}_{a}\right)\right) \\
& \leq C^{\prime}\left(P^{\emptyset A}\left(\widetilde{\theta}_{q}, \widetilde{\theta}_{a}\right)\right)\left(P^{Q A}\left(\widetilde{\theta}_{q}, \widetilde{\theta}_{a}\right)-P^{\emptyset A}\left(\widetilde{\theta}_{q}, \widetilde{\theta}_{a}\right)\right)+C^{\prime}\left(P^{Q \emptyset}\left(\widetilde{\theta}_{q}, \widetilde{\theta}_{a}\right)\right) P^{Q A}\left(\widetilde{\theta}_{q}, \widetilde{\theta}_{a}\right)
\end{aligned}
$$

\footnotetext{
${ }^{19}$ In particular we use that $h^{(Q \emptyset)=(Q A)}\left(g^{(Q \emptyset)=(Q A)}\left(\theta_{a}\right)\right)=\theta_{a}$.
} 
Using now that $\widetilde{\theta}_{q}=g^{(Q \emptyset)=(\emptyset \emptyset)}\left(\widetilde{\theta}_{a}\right)$ verifies $(17)$, and the second line of $(11)$, we have that the type $\left(\widetilde{\theta}_{q}, \widetilde{\theta}_{a}\right)$ prefers $(Q A)$ to $(\emptyset A)$ if

$$
\begin{aligned}
& C^{\prime}\left(P^{Q A}\left(\widetilde{\theta}_{q}, \widetilde{\theta}_{a}\right)\right)\left(P^{Q A}\left(\widetilde{\theta}_{q}, \widetilde{\theta}_{a}\right)-P^{Q \emptyset}\left(\widetilde{\theta}_{q}, \widetilde{\theta}_{a}\right)\right) \\
& \geq C\left(P^{Q A}\left(\widetilde{\theta}_{q}, \widetilde{\theta}_{a}\right)\right)-C\left(P^{\emptyset A}\left(\widetilde{\theta}_{q}, \widetilde{\theta}_{a}\right)\right)-C\left(P^{Q \emptyset}\left(\widetilde{\theta}_{q}, \widetilde{\theta}_{a}\right)\right) \\
& -C^{\prime}\left(P^{\emptyset A}\left(\widetilde{\theta}_{q}, \widetilde{\theta}_{a}\right)\right)\left(P^{Q \emptyset}\left(\widetilde{\theta}_{q}, \widetilde{\theta}_{a}\right)-P^{\emptyset A}\left(\widetilde{\theta}_{q}, \widetilde{\theta}_{a}\right)\right)
\end{aligned}
$$

or

$$
\begin{aligned}
& C^{\prime}\left(P^{Q A}\left(\widetilde{\theta}_{q}, \widetilde{\theta}_{a}\right)\right)\left(P^{Q A}\left(\widetilde{\theta}_{q}, \widetilde{\theta}_{a}\right)-P^{\emptyset A}\left(\widetilde{\theta}_{q}, \widetilde{\theta}_{a}\right)\right) \\
& \geq C\left(P^{Q A}\left(\widetilde{\theta}_{q}, \widetilde{\theta}_{a}\right)\right)-C\left(P^{\emptyset A}\left(\widetilde{\theta}_{q}, \widetilde{\theta}_{a}\right)\right)-C\left(P^{Q \emptyset}\left(\widetilde{\theta}_{q}, \widetilde{\theta}_{a}\right)\right) \\
& -C^{\prime}\left(P^{Q \emptyset}\left(\widetilde{\theta}_{q}, \widetilde{\theta}_{a}\right)\right)\left(P^{\emptyset A}\left(\widetilde{\theta}_{q}, \widetilde{\theta}_{a}\right)-P^{Q \emptyset}\left(\widetilde{\theta}_{q}, \widetilde{\theta}_{a}\right)\right)
\end{aligned}
$$

Note that if $P^{Q \emptyset}\left(\widetilde{\theta}_{q}, \widetilde{\theta}_{a}\right)>P^{\emptyset A}\left(\widetilde{\theta}_{q}, \widetilde{\theta}_{a}\right)$ condition (19) gives that it is sufficient if

$$
\begin{aligned}
-C\left(P^{\emptyset A}\left(\widetilde{\theta}_{q}, \widetilde{\theta}_{a}\right)\right) & \leq C\left(P^{Q \emptyset}\left(\widetilde{\theta}_{q}, \widetilde{\theta}_{a}\right)\right)-C\left(P^{Q A}\left(\widetilde{\theta}_{q}, \widetilde{\theta}_{a}\right)\right) \\
& +C^{\prime}\left(P^{Q A}\left(\widetilde{\theta}_{q}, \widetilde{\theta}_{a}\right)\right)\left(P^{Q A}\left(\widetilde{\theta}_{q}, \widetilde{\theta}_{a}\right)-P^{Q \emptyset}\left(\widetilde{\theta}_{q}, \widetilde{\theta}_{a}\right)\right)
\end{aligned}
$$

and if $P^{Q \emptyset}\left(\widetilde{\theta}_{q}, \widetilde{\theta}_{a}\right) \leq P^{\emptyset A}\left(\widetilde{\theta}_{q}, \widetilde{\theta}_{a}\right)$ condition (20) implies that it is sufficient if

$$
\begin{aligned}
-C\left(P^{Q \emptyset}\left(\widetilde{\theta}_{q}, \widetilde{\theta}_{a}\right)\right) & \leq C\left(P^{\emptyset A}\left(\widetilde{\theta}_{q}, \widetilde{\theta}_{a}\right)\right)-C\left(P^{Q A}\left(\widetilde{\theta}_{q}, \widetilde{\theta}_{a}\right)\right) \\
& +C^{\prime}\left(P^{Q A}\left(\widetilde{\theta}_{q}, \widetilde{\theta}_{a}\right)\right)\left(P^{Q A}\left(\widetilde{\theta}_{q}, \widetilde{\theta}_{a}\right)-P^{\emptyset A}\left(\widetilde{\theta}_{q}, \widetilde{\theta}_{a}\right)\right)
\end{aligned}
$$

Convexity of $C$ gives that the right hand side of the previous two conditions is always positive so if the type $\left(\widetilde{\theta}_{q}, \widetilde{\theta}_{a}\right)$ verifies $\widetilde{\theta}_{q}=g^{(Q \emptyset)=(\emptyset \emptyset)}\left(\widetilde{\theta}_{a}\right)$ we must have that $\left(\widetilde{\theta}_{q}, \widetilde{\theta}_{a}\right)$ prefers $Q A$ to $\emptyset A$ which implies $\widetilde{\theta}_{q} \geq g^{(\emptyset A)=(Q A)}\left(\widetilde{\theta}_{a}\right)$ by definition of $g^{(\emptyset A)=(Q A)}\left(\theta_{a}\right)$.

Remark 4 If $g^{(\emptyset A)=(Q A)}\left(\theta_{a}\right) \neq g^{(A A)=(\emptyset A)}\left(\theta_{a}\right)$ for some $\theta_{a} \neq 0$ then

$$
\begin{aligned}
& g^{(\emptyset A)=(Q A)}\left(\theta_{a}\right)<g^{(A A)=(Q A)}\left(\theta_{a}\right)<g^{(A A)=(\emptyset A)}\left(\theta_{a}\right) \text { or } \\
& g^{(A A)=(\emptyset A)}\left(\theta_{a}\right)<g^{(A A)=(Q A)}\left(\theta_{a}\right)<g^{(\emptyset A)=(Q A)}\left(\theta_{a}\right)
\end{aligned}
$$

Moreover, 1) if $L_{Q \rightarrow \emptyset} \leq L_{\emptyset \rightarrow A}$ then the first line holds for any $\theta_{a}>0$, and 2) if $L_{Q \rightarrow \emptyset}>L_{\emptyset \rightarrow A}$ then the second line holds for sufficiently small $\theta_{a}$.

Assume first that $g^{(\emptyset A)=(Q A)}\left(\theta_{a}\right) \leq g^{(A A)=(\emptyset A)}\left(\theta_{a}\right)$ so every type with $\theta_{q}=g^{(\emptyset A)=(Q A)}\left(\theta_{a}\right)$ weakly prefers $\emptyset A$ to $Q A$ and strictly prefers $A A$ to $\emptyset A$ so it must be that $A A$ is strictly 
prefer to $Q A$ which implies that $\theta_{q}<g^{(A A)=(Q A)}\left(\theta_{a}\right)$ or, by assumption, $g^{(\emptyset A)=(Q A)}\left(\theta_{a}\right)<$ $g^{(A A)=(Q A)}\left(\theta_{a}\right)$. Now take $\theta_{q}=g^{(A A)=(Q A)}\left(\theta_{a}\right)$, so $A A$ is weakly preferred to $Q A$, and since $g^{(\emptyset A)=(Q A)}\left(\theta_{a}\right)<\theta_{q}$ we must have that $Q A$ is preferred to $\emptyset A$ and, therefore, $A A$ is preferred to $\emptyset A$ which implies that $\theta_{q}<g^{(A A)=(\emptyset A)}\left(\theta_{a}\right)$, and therefore $g^{(A A)=(Q A)}\left(\theta_{a}\right)<g^{(A A)=(\emptyset A)}\left(\theta_{a}\right)$. This proves the first line. The second line follows by assuming that $g^{(\emptyset A)=(Q A)}\left(\theta_{a}\right)>$ $g^{(A A)=(\emptyset A)}\left(\theta_{a}\right)$ and using the same arguments.

For last part we have that it is sufficient if $g^{(\emptyset A)=(Q A)}\left(\theta_{a}\right)<g^{(A A)=(Q A)}\left(\theta_{a}\right)$ when $L_{Q \rightarrow \emptyset} \leq$ $L_{Q \rightarrow A} \leq L_{\emptyset \rightarrow A}$. Using (18) we have that every type with $\widetilde{\theta}_{q}=g^{(\emptyset A)=(Q A)}\left(\widetilde{\theta}_{a}\right)$ verifies

$$
\begin{aligned}
& \widetilde{\theta}_{q} \frac{\operatorname{Pr}\left(p i v_{Q} \mid q\right)}{2} P^{Q A}\left(\widetilde{\theta}_{q}, \widetilde{\theta}_{a}\right)-\widetilde{\theta}_{a} \frac{\operatorname{Pr}\left(p i v_{Q} \mid a\right)}{2}\left(1-P^{Q A}\left(\widetilde{\theta}_{q}, \widetilde{\theta}_{a}\right)\right) \\
& =C\left(P^{Q A}\left(\widetilde{\theta}_{q}, \widetilde{\theta}_{a}\right)\right)-C\left(P^{\emptyset A}\left(\widetilde{\theta}_{q}, \widetilde{\theta}_{a}\right)\right) \\
& -C^{\prime}\left(P^{\emptyset A}\left(\widetilde{\theta}_{q}, \widetilde{\theta}_{a}\right)\right)\left(P^{Q A}\left(\widetilde{\theta}_{q}, \widetilde{\theta}_{a}\right)-P^{\emptyset A}\left(\widetilde{\theta}_{q}, \widetilde{\theta}_{a}\right)\right)
\end{aligned}
$$

By concavity of $C$ and the fact that $P^{\emptyset A}\left(\widetilde{\theta}_{q}, \widetilde{\theta}_{a}\right)<P^{Q A}\left(\widetilde{\theta}_{q}, \widetilde{\theta}_{a}\right)$ we have that the right hand side of (21) is negative

$$
\frac{\widetilde{\theta}_{q}}{\widetilde{\theta}_{a}}<L_{Q \rightarrow \emptyset} \frac{\left(1-P^{Q A}\left(\widetilde{\theta}_{q}, \widetilde{\theta}_{a}\right)\right)}{P^{Q A}\left(\widetilde{\theta}_{q}, \widetilde{\theta}_{a}\right)} \leq L_{Q \rightarrow A} \frac{\left(1-P^{Q A}\left(\widetilde{\theta}_{q}, \widetilde{\theta}_{a}\right)\right)}{P^{Q A}\left(\widetilde{\theta}_{q}, \widetilde{\theta}_{a}\right)}
$$

where the inequality on the right follows by assumption of $L_{Q \rightarrow \emptyset} \leq L_{Q \rightarrow A}$. Using now (3), (11) and (14) we have that $g^{(A A)=(Q A)}\left(\theta_{a}\right)$ is defined as

$$
\theta_{a} \frac{\operatorname{Pr}\left(\operatorname{piv}_{Q} \cup p i v_{A} \mid a\right)}{2}=\mathcal{L}\left(P^{Q A}\left(g^{(A A)=(Q A)}\left(\theta_{a}\right), \theta_{a}\right)\right)
$$

which implies that $g^{(A A)=(Q A)}\left(\theta_{a}\right)$ is strictly concave with

$$
\frac{\partial g^{(A A)=(Q A)}\left(\theta_{a}\right)}{\partial \theta_{a}}=L_{Q \rightarrow A} \frac{1-P^{Q A}\left(g^{(A A)=(Q A)}\left(\theta_{a}\right), \theta_{a}\right)}{P^{Q A}\left(g^{(A A)=(Q A)}\left(\theta_{a}\right), \theta_{a}\right)}
$$

Using properties of concave functions we have that $\frac{g^{(A A)=(Q A)}\left(\theta_{a}\right)}{\theta_{a}}>L_{Q \rightarrow A} \frac{1-P^{Q A}\left(g^{(A A)=(Q A)}\left(\theta_{a}\right), \theta_{a}\right)}{P^{Q A}\left(g^{(A A)=(Q A)}\left(\theta_{a}\right), \theta_{a}\right)}$ which implies that, for the type that verifies $\widetilde{\theta}_{q}=g^{(\emptyset A)=(Q A)}\left(\widetilde{\theta}_{a}\right)$ using the condition (22): $\frac{g^{(A A)=(Q A)}\left(\widetilde{\theta}_{a}\right)}{\widetilde{\theta}_{a}} \frac{P^{Q A}\left(g^{(A A)=(Q A)}\left(\widetilde{\theta}_{a}\right), \widetilde{\theta}_{a}\right)}{1-P^{Q A}\left(g^{(A A)=(Q A)}\left(\widetilde{\theta}_{a}\right), \widetilde{\theta}_{a}\right)}>\frac{\widetilde{\theta}_{q}}{\widetilde{\theta}_{a}} \frac{P^{Q A}\left(\widetilde{\theta}_{q}, \widetilde{\theta}_{a}\right)}{1-P^{Q A}\left(\widetilde{\theta}_{q}, \widetilde{\theta}_{a}\right)}$. Note now that $\frac{x}{y} \frac{P^{Q A}(x, y)}{1-P^{Q A}(x, y)}$ is increasing in $x$ which implies that $g^{(A A)=(Q A)}\left(\widetilde{\theta}_{a}\right)>\widetilde{\theta}_{q}=g^{(\emptyset A)=(Q A)}\left(\widetilde{\theta}_{a}\right)$.

Finally, note that (21) implies that

$$
\frac{\partial g^{(\emptyset A)=(Q A)}\left(\widetilde{\theta}_{a}\right)}{\partial \widetilde{\theta}_{a}}=\frac{\frac{\operatorname{Pr}\left(p i v_{Q} \cup p i v_{A} \mid a\right)}{2}\left(1-P^{Q A}\left(\widetilde{\theta}_{q}, \widetilde{\theta}_{a}\right)\right)-\frac{\operatorname{Pr}\left(p i v_{A} \mid a\right)}{2}\left(1-P^{\emptyset A}\left(\widetilde{\theta}_{q}, \widetilde{\theta}_{a}\right)\right)}{\frac{\operatorname{Pr}\left(\operatorname{piv}_{Q} \cup p i v_{A} \mid q\right)}{2} P^{Q A}\left(\widetilde{\theta}_{q}, \widetilde{\theta}_{a}\right)-\frac{\operatorname{Pr}\left(p i v_{A} \mid q\right)}{2} P^{\emptyset A}\left(\widetilde{\theta}_{q}, \widetilde{\theta}_{a}\right)}
$$


so when $\widetilde{\theta}_{a} \rightarrow 0$ we have that $\frac{\partial g^{(\emptyset A)=(Q A)}\left(\widetilde{\theta}_{a}\right)}{\partial \widetilde{\theta}_{a}} \rightarrow L_{Q \rightarrow \emptyset}$ while $\frac{\partial g^{(A A)=(Q A)}\left(\theta_{a}\right)}{\partial \theta_{a}} \rightarrow L_{Q \rightarrow A}$; then if $L_{Q \rightarrow \emptyset}>L_{Q \rightarrow A}>L_{\emptyset \rightarrow A}$ we must have then $g^{(\emptyset A)=(Q A)}\left(\widetilde{\theta}_{a}\right)>g^{(A A)=(Q A)}\left(\theta_{a}\right)$ for sufficiently small $\theta_{a}$.

Remark 5 If $L_{Q \rightarrow \emptyset} \leq L_{\emptyset \rightarrow A}$ then $g^{(\emptyset A)=(\emptyset \emptyset)}\left(\theta_{a}\right) \geq g^{(Q \emptyset)=(\emptyset \emptyset)}\left(\theta_{a}\right)$ and if $L_{Q \rightarrow \emptyset}>L_{\emptyset \rightarrow A}$ then there is some $\theta_{a}$ sufficiently small such that $g^{(\emptyset A)=(\emptyset \emptyset)}\left(\theta_{a}\right)<g^{(Q \emptyset)=(\emptyset \emptyset)}\left(\theta_{a}\right)$.

Note that $(16)$ gives $L_{\emptyset \rightarrow A} \frac{P^{(\emptyset A)}\left(g^{(\emptyset A)=(\emptyset \emptyset)}\left(\theta_{a}\right), \theta_{a}\right)}{1-P^{(\emptyset A)}\left(g^{(\emptyset A)=(\emptyset \emptyset)}\left(\theta_{a}\right), \theta_{a}\right)} \equiv \frac{\partial g^{(\emptyset A)=(\emptyset \emptyset)}\left(\theta_{a}\right)}{\partial \theta_{a}}$ and $(17)$ gives $\frac{\partial g^{(Q \emptyset)=(\emptyset \emptyset)}\left(\theta_{a}\right)}{\partial \theta_{a}}=$ $L_{Q \rightarrow \emptyset} \frac{1-P^{Q \emptyset}\left(g^{(Q \emptyset)=(\emptyset \emptyset)}\left(\theta_{a}\right), \theta_{a}\right)}{P^{Q \emptyset}\left(g^{(Q \emptyset)=(\emptyset \emptyset)}\left(\theta_{a}\right), \theta_{a}\right)}$ which implies that $\theta_{a} \rightarrow 0$ we must have $\frac{\partial g^{(\emptyset A)=(\emptyset \emptyset)}\left(\theta_{a}\right)}{\partial \theta_{a}} \rightarrow L_{\emptyset \rightarrow A}$ and $\frac{\partial g^{(Q \emptyset)=(\emptyset \emptyset)}\left(\theta_{a}\right)}{\partial \theta_{a}} \rightarrow L_{Q \rightarrow \emptyset}$. Using that $g^{(\emptyset A)=(\emptyset \emptyset)}\left(\theta_{a}\right) \rightarrow 0$ and $g^{(Q \emptyset)=(\emptyset \emptyset)}\left(\theta_{a}\right) \rightarrow 0$ when $\theta_{a} \rightarrow 0$, if $L_{Q \rightarrow \emptyset}>L_{\emptyset \rightarrow A}$ there is some $\theta_{a}$ sufficiently small such that $g^{(\emptyset A)=(\emptyset \emptyset)}\left(\theta_{a}\right)<g^{(Q \emptyset)=(\emptyset \emptyset)}\left(\theta_{a}\right)$.

We are now ready to provide the proof of Proposition 1

Proof. Using the cutoff functions described previously let's first define the following group of voters. ${ }^{20}$ The set of strong supporters of each candidate are defined by

$$
\begin{aligned}
& \mathcal{S S}^{A} \equiv\left\{\theta \in[0,1]^{2}: \theta_{q} \leq \min \left\{g^{(A A)=(\emptyset A)}\left(\theta_{a}\right), g^{(A A)=(Q A)}\left(\theta_{a}\right)\right\}\right\} \\
& \mathcal{S S}^{Q} \equiv\left\{\theta \in[0,1]^{2}: \theta_{q} \geq \max \left\{g^{(Q Q)=(Q \emptyset)}\left(\theta_{a}\right), g^{(Q Q)=(Q A)}\left(\theta_{a}\right)\right\}\right\}
\end{aligned}
$$

The sets of weak supporters are defined $\mathrm{as}^{21}$ :

$$
\begin{aligned}
& \mathcal{W S}^{A} \equiv\left\{\theta \in[0,1]^{2}: g^{(A A)=(\emptyset A)}\left(\theta_{a}\right)<\theta_{q} \leq g^{(\emptyset A)=(\emptyset \emptyset)}\left(\theta_{a}\right), \theta_{a} \geq h^{(\emptyset A)=(Q A)}\left(\theta_{q}\right)\right\} \\
& \mathcal{W S}^{Q} \equiv\left\{\theta \in[0,1]^{2}: g^{(Q \emptyset)=(\emptyset \emptyset)}\left(\theta_{a}\right) \leq \theta_{q}<g^{(Q Q)=(Q \emptyset)}\left(\theta_{a}\right), \theta_{a} \leq h^{(Q \emptyset)=(Q A)}\left(\theta_{q}\right)\right\}
\end{aligned}
$$

The case of independents and abstainers involves the function $g^{(Q A)=(\emptyset \emptyset)}\left(\theta_{a}\right)$ or $h^{(Q A)=(\emptyset \emptyset)}\left(\theta_{q}\right)$ depending on which one is properly defined. If $g^{(Q A)=(\emptyset \emptyset)}\left(\theta_{a}\right)$ is well defined, the set of abstainers is

$$
\mathcal{A} \equiv\left\{\theta \in[0,1]^{2}:\left[\begin{array}{c}
\theta_{q} \leq g^{(Q A)=(\emptyset \emptyset)}\left(\theta_{a}\right) \\
, g^{(\emptyset A)=(\emptyset \emptyset)}\left(\theta_{a}\right)<\theta_{q}<g^{(Q \emptyset)=(\emptyset \emptyset)}\left(\theta_{a}\right)
\end{array}\right]\right\}
$$

while if $h^{(Q A)=(\emptyset \emptyset)}\left(\theta_{q}\right)$ is well defined, the set of abstainers is

$$
\mathcal{A} \equiv\left\{\theta \in[0,1]^{2}:\left[\begin{array}{c}
\theta_{a} \leq h^{(Q A)=(\emptyset \emptyset)}\left(\theta_{q}\right) \\
, g^{(\emptyset A)=(\emptyset \emptyset)}\left(\theta_{a}\right)<\theta_{q}<g^{(Q \emptyset)=(\emptyset \emptyset)}\left(\theta_{a}\right)
\end{array}\right]\right\}
$$

Independents are obviously defined as the complement of all these groups in $[0,1]^{2}$.

The first part of the characterization involves showing that the reduced set of cutoff functions that describe each one of these sets are necessary and sufficient. We focus on the

\footnotetext{
${ }^{20}$ Since its measure is zero we can assign types that are indifferent to any of the groups that provide the same expected utility.

${ }^{21}$ Strictly speaking $h^{(\emptyset A)=(Q A)}\left(\theta_{q}\right)$ is not necessary since the function $g^{(\emptyset A)=(Q A)}\left(\theta_{a}\right)$ is well defined. We provide that representation in order to maintain the symmetric structure.
} 
sets of $A$ weak supporters $\left(\mathcal{W S}^{A}\right)$, abstainers $(\mathcal{A})$, and $A$ strong supporters $\left(\mathcal{S} \mathcal{S}^{A}\right)$. For these sets be check that voters are maximizing expected utility by selecting those strategies and using the optimal information. The sets of $Q$ strong supporters $\left(\mathcal{S S}^{Q}\right)$ and $Q$ weak supporters $\left(\mathcal{W S}^{Q}\right)$ can be analyzed by reversing the order of $A$ and $Q$ and the signals $s_{a}$ and $s_{q}$ in the analysis. Finally the set of independents $(\mathcal{I})$ can be defined as the complement of the remaining sets of voters.

\section{$\mathcal{W S}^{A}$ is well defined by (26)}

Using Remarks 2 and 3 we have $g^{(\emptyset A)=(Q A)}\left(\theta_{a}\right)<g^{(Q \emptyset)=(\emptyset \emptyset)}\left(\theta_{a}\right)<g^{(Q \emptyset)=(Q Q)}\left(\theta_{a}\right)$ so if $\theta_{q} \leq g^{(\emptyset A)=(Q A)}\left(\theta_{a}\right)$, the strategy $\emptyset A$ is preferred to the strategy $Q A$ and the strategy $\emptyset \emptyset$ is preferred to the strategies $Q \emptyset$ and $Q Q$. Now using that if $\theta_{q} \leq g^{(\emptyset A)=(\emptyset \emptyset)}\left(\theta_{a}\right)$, the strategy $\emptyset A$ is preferred to the strategy $\emptyset \emptyset$, and by the previous line it is also preferred to the strategies $Q \emptyset$ and $Q Q$. Finally, if $\theta_{q}>g^{(A A)=(\emptyset A)}\left(\theta_{a}\right)$, the strategy $\emptyset A$ is preferred to the strategy $A A$.

Using now Remark 4 we have that $L_{Q \rightarrow \emptyset} \leq L_{Q \rightarrow A} \leq L_{\emptyset \rightarrow A}$ implies $g^{(\emptyset A)=(Q A)}\left(\theta_{a}\right) \leq$ $g^{(A A)=(\emptyset A)}\left(\theta_{a}\right)$ so the set $\left\{\theta \in[0,1]^{2}: g^{(A A)=(\emptyset A)}\left(\theta_{a}\right)<\theta_{q} \leq g^{(\emptyset A)=(\emptyset \emptyset)}\left(\theta_{a}\right)\right\}=\emptyset$ which implies that $\mathcal{W S}^{A}=\emptyset$. On the other hand, Remark 4 and the fact that $h$ is the mnemonic for the inverse of $g$, we have that $L_{Q \rightarrow \emptyset}>L_{Q \rightarrow A}>L_{\emptyset \rightarrow A}$, implies that for sufficiently small $\theta_{a}$, $g^{(\emptyset A)=(Q A)}\left(\theta_{a}\right)>g^{(A A)=(Q A)}\left(\theta_{a}\right)>g^{(A A)=(\emptyset A)}\left(\theta_{a}\right)$ and $\mathcal{W S}^{A} \neq \emptyset$.

\section{$\mathcal{A}$ is well defined by (27) and (28)}

We want to show that the set of abstainers defined either as in (27) or in (28) is well defined whenever $g^{(Q A)=(\emptyset \emptyset)}\left(\theta_{a}\right)$ and/or $h^{(Q A)=(\emptyset \emptyset)}\left(\theta_{a}\right)$ are well defined.

It is immediate to see that $g^{(\emptyset A)=(\emptyset \emptyset)}\left(\theta_{a}\right)<\theta_{q}<g^{(Q \emptyset)=(\emptyset \emptyset)}\left(\theta_{a}\right)$ implies that $\emptyset \emptyset$ is preferred to any strategy followed by a weak supporter. Remark 1 which implies $g^{(\emptyset A)=(\emptyset \emptyset)}\left(\theta_{a}\right)>$ $g^{(A A)=(\emptyset A)}\left(\theta_{a}\right)$ and Remark 2 which implies $g^{(Q \emptyset)=(\emptyset \emptyset)}\left(\theta_{a}\right)<g^{(Q \emptyset)=(Q Q)}\left(\theta_{a}\right)$ we have that $g^{(A A)=(\emptyset A)}\left(\theta_{a}\right)<\theta_{q}<g^{(Q \emptyset)=(Q Q)}\left(\theta_{a}\right)$ implies that $\emptyset A$ is preferred to $A A$ and $Q \emptyset$ is preferred to $Q Q$, so using the previous argument we have that $\emptyset \emptyset$ is preferred to any strategy followed by a strong supporter.

Using Remark 5 we have that if $L_{Q \rightarrow \emptyset} \leq L_{\emptyset \rightarrow A}$ then $\mathcal{A}=\emptyset$ and if $L_{Q \rightarrow \emptyset}>L_{\emptyset \rightarrow A}$ we need to show that that abstaining without information is preferred to voting following the signal. First we are going to prove that the cutoff functions are well defined so the sets can be properly defined. Using $(3),(11),(14)$, and the function $\mathcal{L}$ we define $g^{(Q A)=(\emptyset \emptyset)}\left(\theta_{a}\right)$ and $h^{(Q A)=(\emptyset \emptyset)}\left(\theta_{q}\right)$, whenever possible, as

$$
\begin{aligned}
& \mathcal{L}\left(P^{Q A}\left(g^{(Q A)=(\emptyset \emptyset)}\left(\theta_{a}\right), \theta_{a}\right)\right)=g^{(Q A)=(\emptyset \emptyset)}\left(\theta_{a}\right) \frac{\operatorname{Pr}\left(\text { piv }_{A} \mid q\right)}{2}+\theta_{a} \frac{\operatorname{Pr}\left(\text { piv }_{Q} \mid a\right)}{2} \\
& \mathcal{L}\left(P^{Q A}\left(\theta_{q}, h^{(Q A)=(\emptyset \emptyset)}\left(\theta_{q}\right)\right)\right)=\theta_{q} \frac{\operatorname{Pr}\left(\text { piv }_{A} \mid q\right)}{2}+h^{(Q A)=(\emptyset \emptyset)}\left(\theta_{q}\right) \frac{\operatorname{Pr}\left(\text { piv }_{Q} \mid a\right)}{2}
\end{aligned}
$$

and using the implicit function theorem we have that $g^{(Q A)=(\emptyset \emptyset)}\left(\theta_{a}\right)$ is well defined when $\frac{\operatorname{Pr}\left(p i v_{A} \mid q\right)}{\operatorname{Pr}\left(\operatorname{piv}_{Q} \cup p i v_{A} \mid q\right)} \neq P^{Q A}\left(g^{(Q A)=(\emptyset \emptyset)}\left(\theta_{a}\right), \theta_{a}\right)$ and $h^{(Q A)=(\emptyset \emptyset)}\left(\theta_{q}\right)$ is well defined when $\frac{\operatorname{Pr}\left(p i v_{A} \mid a\right)}{\operatorname{Pr}\left(\operatorname{piv}_{Q} \cup p i v_{A} \mid a\right)} \neq$ $1-P^{Q A}\left(\theta_{q}, h^{(Q A)=(\emptyset \emptyset)}\left(\theta_{q}\right)\right)$.

First note that if the left hand side of any of the equations in (29) is bigger than the right hand side, we have that the strategy $Q A$ is preferred to $\emptyset \emptyset$. Assume that $\emptyset \emptyset$ is indifferent to 
$Q A$ so we must have the first line of $(29)$ is equivalent to

$$
\begin{aligned}
& \frac{\theta_{q}}{\theta_{a}} \frac{\operatorname{Pr}\left(\text { piv }_{Q} \cup \text { piv }_{A} \mid q\right)}{\operatorname{Pr}\left(\text { piv }_{Q} \cup \text { piv }_{A} \mid a\right)}\left(P^{Q A}\left(\theta_{q}, \theta_{a}\right)-\frac{\operatorname{Pr}\left(\text { piv }_{A} \mid q\right)}{\operatorname{Pr}\left(\text { piv }_{Q} \cup p i v_{A} \mid q\right)}\right) \\
& =\left(\left(1-P^{Q A}\left(\theta_{q}, \theta_{a}\right)\right)-\frac{\operatorname{Pr}\left(\text { piv }_{A} \mid a\right)}{\operatorname{Pr}\left(\text { piv }_{Q} \cup \text { piv }_{A} \mid a\right)}\right)+C\left(P^{Q A}\left(\theta_{q}, \theta_{a}\right)\right)
\end{aligned}
$$

Assume that $\left(\theta_{q}, \theta_{a}\right)$ verifies that $P^{Q A}\left(\theta_{q}, \theta_{a}\right) \leq \frac{\operatorname{Pr}\left(p i v_{A} \mid q\right)}{\operatorname{Pr}\left(p i v_{Q} \cup p i v_{A} \mid q\right)}$ so we must have that

$$
\begin{aligned}
& \frac{\theta_{q}}{\theta_{a}} \frac{\operatorname{Pr}\left(\text { piv }_{Q} \cup \text { piv }_{A} \mid q\right)}{\operatorname{Pr}\left(\text { piv }_{Q} \cup \operatorname{piv}_{A} \mid a\right)}\left(P^{Q A}\left(\theta_{q}, \theta_{a}\right)-\frac{\operatorname{Pr}\left(\text { piv }_{A} \mid q\right)}{\operatorname{Pr}\left(\text { piv }_{Q} \cup p i v_{A} \mid q\right)}\right) \\
& >\left(\left(1-P^{Q A}\left(\theta_{q}, \theta_{a}\right)\right)-\frac{\operatorname{Pr}\left(\text { piv }_{A} \mid a\right)}{\operatorname{Pr}\left(\text { piv }_{Q} \cup \operatorname{piv}_{A} \mid a\right)}\right)
\end{aligned}
$$

Recalling that (5), (7) and $L_{Q \rightarrow \emptyset}>L_{\emptyset \rightarrow A}$ imply that $L_{\emptyset \rightarrow A}<\frac{\theta_{q}}{\theta_{a}}<L_{Q \rightarrow \emptyset}$ is necessary for abstention we have that

$$
\begin{aligned}
& P^{Q A}\left(\theta_{q}, \theta_{a}\right)>\frac{\frac{\operatorname{Pr}\left(\text { piv }_{Q} \cup p i v_{A} \mid a\right)}{\operatorname{Pr}\left(\text { piv }_{Q} \cup p i v_{A} \mid q\right)}}{\frac{\operatorname{Pr}\left(\text { piv }_{A} \mid a\right)}{\operatorname{Pr}\left(\text { piv }_{A} \mid q\right)}+\frac{\operatorname{Pr}\left(\text { piv }_{Q} \cup p i v_{A} \mid a\right)}{\operatorname{Pr}\left(\text { piv }_{Q} \cup p i v_{A} \mid q\right)}}
\end{aligned}
$$

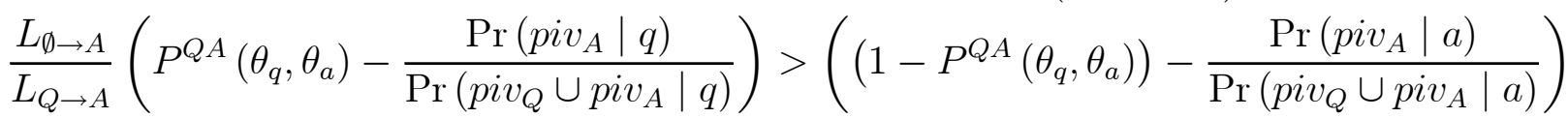

where the second line follows since we assume $P^{Q A}\left(\theta_{q}, \theta_{a}\right)-\frac{\operatorname{Pr}\left(p i v_{A} \mid q\right)}{\operatorname{Pr}\left(\operatorname{piv}_{Q} \cup p i v_{A} \mid q\right)} \leq 0$. Using again the assumption that $P^{Q A}\left(\theta_{q}, \theta_{a}\right) \leq \frac{\operatorname{Pr}\left(p i v_{A} \mid q\right)}{\operatorname{Pr}\left(p i v_{Q} \cup p i v_{A} \mid q\right)}$ we have that (30) implies that

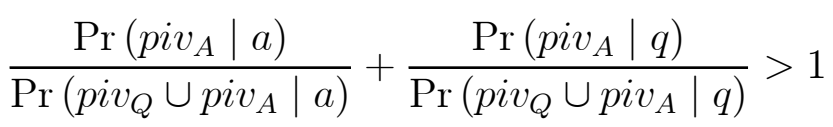

Therefore, if $\frac{\operatorname{Pr}\left(p i v_{A} \mid q\right)}{\operatorname{Pr}\left(\operatorname{piv}_{Q} \cup p i v_{A} \mid q\right)} \leq \frac{\operatorname{Pr}\left(\operatorname{piv}_{Q} \mid a\right)}{\operatorname{Pr}\left(\operatorname{piv}_{Q} \cup p i v_{A} \mid a\right)}$ every type that it is indifferent between $\emptyset \emptyset$ and $Q A$ verifies that $P^{Q A}\left(\theta_{q}, \theta_{a}\right)>\frac{\operatorname{Pr}\left(p i v_{A} \mid q\right)}{\operatorname{Pr}\left(\operatorname{piv}_{Q} \cup p i v_{A} \mid q\right)}$ whenever $L_{Q \rightarrow \emptyset}>L_{\emptyset \rightarrow A}$. Now assume that $\frac{\operatorname{Pr}\left(p i v_{A} \mid a\right)}{\operatorname{Pr}\left(\operatorname{piv}_{Q} \cup p i v_{A} \mid a\right)}+\frac{\operatorname{Pr}\left(p i v_{A} \mid q\right)}{\operatorname{Pr}\left(\operatorname{piv}_{Q} \cup p i v_{A} \mid q\right)}>1$ and we have that the first line of (30) implies that

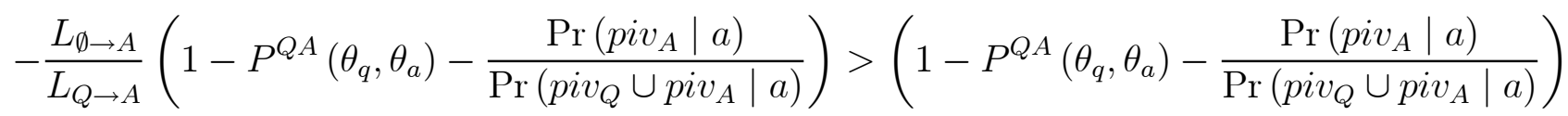

so we must have that $\left(1-P^{Q A}\left(\theta_{q}, \theta_{a}\right)\right)<\frac{\operatorname{Pr}\left(p_{i} v_{A} \mid a\right)}{\operatorname{Pr}\left(\text { piv }_{Q} \cup p i v_{A} \mid a\right)}$.

Recalling that $g^{(Q A)=(\emptyset \emptyset)}\left(\theta_{a}\right)$ is well defined when $\frac{\operatorname{Pr}\left(\operatorname{piv}_{A} \mid q\right)}{\operatorname{Pr}\left(\operatorname{piv}_{Q} \cup p i v_{A} \mid q\right)} \neq P^{Q A}\left(g^{(Q A)=(\emptyset \emptyset)}\left(\theta_{a}\right), \theta_{a}\right)$ and $h^{(Q A)=(\emptyset \emptyset)}\left(\theta_{q}\right)$ is well defined when $\frac{\operatorname{Pr}\left(p i v_{A} \mid a\right)}{\operatorname{Pr}\left(\operatorname{piv}_{Q} \cup p i v_{A} \mid a\right)} \neq 1-P^{Q A}\left(\theta_{q}, h^{(Q A)=(\emptyset \emptyset)}\left(\theta_{q}\right)\right)$ we have 
that $\frac{\operatorname{Pr}\left(p i v_{A} \mid a\right)}{\operatorname{Pr}\left(\operatorname{piv}_{Q} \cup p i v_{A} \mid a\right)}+\frac{\operatorname{Pr}\left(p i v_{A} \mid q\right)}{\operatorname{Pr}\left(\operatorname{piv}_{Q} \cup p i v_{A} \mid q\right)} \leq 1$ is sufficient to apply the implicit function theorem for $g^{(Q A)=(\emptyset \emptyset)}\left(\theta_{a}\right)$ and $\frac{\operatorname{Pr}\left(p i v_{A} \mid a\right)}{\operatorname{Pr}\left(\operatorname{piv}_{Q} \cup p i v_{A} \mid a\right)}+\frac{\operatorname{Pr}\left(p i v_{A} \mid q\right)}{\operatorname{Pr}\left(\operatorname{piv}_{Q} \cup p i v_{A} \mid q\right)}>1$ is sufficient to apply the implicit function theorem for $h^{(Q A)=(\emptyset \emptyset)}\left(\theta_{q}\right)$.

To finish the proof that $L_{Q \rightarrow \emptyset}>L_{\emptyset \rightarrow A}$ is sufficient for $\mathcal{A}$ to be non empty we use that, 1) Lemma 2 gives that when $L_{Q \rightarrow \emptyset}>L_{\emptyset \rightarrow A}$ there is a sufficient small $\theta_{a}$ such that $\emptyset \emptyset$ is preferred to being independent, and 2) Remark 5 gives that $L_{Q \rightarrow \emptyset}>L_{\emptyset \rightarrow A}$ there is a sufficient small $\theta_{a}$ such that $g^{(\emptyset A)=(\emptyset \emptyset)}\left(\theta_{a}\right)<g^{(Q \emptyset)=(\emptyset \emptyset)}\left(\theta_{a}\right)$.

\section{$\mathcal{S S}^{A}$ is well defined by (25)}

We divide the proof in two: the case $L_{Q \rightarrow \emptyset} \leq L_{\emptyset \rightarrow A}$ and $L_{\emptyset \rightarrow A}<L_{Q \rightarrow \emptyset}$.

Using Remark 4 we have that when $L_{Q \rightarrow \emptyset} \leq L_{Q \rightarrow A} \leq L_{\emptyset \rightarrow A}$ the only relevant comparison is between $A A$ and $Q A$ since $\emptyset A$ is not going to be used in equilibrium. In terms of the cutoffs functions by Remark 4 we have that $g^{(A A)=(Q A)}\left(\theta_{a}\right) \leq g^{(A A)=(\emptyset A)}\left(\theta_{a}\right)$ which implies that $\mathcal{S S}^{A} \equiv\left\{\theta \in[0,1]^{2}: \theta_{q} \leq g^{(A A)=(Q A)}\left(\theta_{a}\right)\right\}$.

We focus now on the second case, $L_{\emptyset \rightarrow A}<L_{Q \rightarrow \emptyset}$. Since $\frac{\partial \Delta \mathcal{U}^{i}\left((A A),(\emptyset A) \mid \theta_{q}, \theta_{a}\right)}{\partial \theta_{q}}<0$, if $\theta_{q}<$ $g^{(A A)=(\emptyset A)}\left(\theta_{a}\right)$ the strategy $A A$ is preferred to the strategy $(\emptyset A)$. On the other hand, since $\frac{\partial \Delta \mathcal{U}^{i}\left((\emptyset A),(\emptyset \emptyset) \mid \theta_{q}, \theta_{a}\right)}{\partial \theta_{q}}<0$, if $\theta_{q}<g^{(\emptyset A)=(\emptyset \emptyset)}\left(\theta_{a}\right)$ the strategy $(\emptyset A)$ is preferred to the strategy $(\emptyset \emptyset)$. Using Remark 1 if a type prefers $A A$ to the strategy $\emptyset A\left(\theta_{q}<g^{(A A)=(\emptyset A)}\left(\theta_{a}\right)\right)$ that type also prefers the strategy $A A$ to the strategy $\emptyset \emptyset$. It is easy to see that $\frac{\partial \Delta \mathcal{U}^{i}\left((A A),(Q A) \mid \theta_{q}, \theta_{a}\right)}{\partial \theta_{q}}<0$ so if $\theta_{q}<g^{(A A)=(Q A)}\left(\theta_{a}\right)$ the strategy $A A$ is preferred to the strategy $Q A$. Putting all together a type that verifies $\theta_{q}<\min \left\{g^{(A A)=(Q A)}\left(\theta_{a}\right), g^{(A A)=(\emptyset A)}\left(\theta_{a}\right)\right\}$ prefers the strategy $A A$ to $Q A, \emptyset A$ and $\emptyset \emptyset$.

Since $\frac{\partial \Delta \mathcal{U}^{i}\left((\emptyset \emptyset),(Q \emptyset) \mid \theta_{q}, \theta_{a}\right)}{\partial \theta_{q}}<0$, if $\theta_{q}<g^{(Q \emptyset)=(\emptyset \emptyset)}\left(\theta_{a}\right)$, the strategy $\emptyset \emptyset$ is preferred to the strategy $Q \emptyset$. On the other hand, since $\frac{\partial \Delta \mathcal{U}^{i}\left((Q \emptyset),(Q Q) \mid \theta_{q}, \theta_{a}\right)}{\partial \theta_{q}}<0$, if $\theta_{q}<g^{(Q \emptyset)=(Q Q)}\left(\theta_{a}\right)$ the strategy $Q \emptyset$ is preferred to the strategy $Q Q$. Using Remark 2 we have that a if $\theta_{q}<$ $g^{(Q \emptyset)=(\emptyset \emptyset)}\left(\theta_{a}\right)$ then a type that prefers $\emptyset \emptyset$ to the strategy $Q \emptyset$ also prefers the strategy $\emptyset \emptyset$ to the strategy $Q Q$.

By Remark 4 we have that $L_{\emptyset \rightarrow A}<L_{Q \rightarrow \emptyset}$ implies that $g^{(A A)=(\emptyset A)}\left(\theta_{a}\right)<g^{(A A)=(Q A)}\left(\theta_{a}\right)<$ $g^{(\emptyset A)=(Q A)}\left(\theta_{a}\right)$ and by Remark 3 we have that $g^{(\emptyset A)=(Q A)}\left(\theta_{a}\right)<g^{(Q \emptyset)=(\emptyset \emptyset)}\left(\theta_{a}\right)$. The previous two paragraphs give the result.

It is easy to verify that the sets $\mathcal{S S}^{A}, \mathcal{S S}^{Q}, \mathcal{W} \mathcal{S}^{A}, \mathcal{W} \mathcal{S}^{Q}, \mathcal{A}$ and $\mathcal{I}$ cover all types in $[0,1]^{2}$ without intersecting each other. This finishes the description of equilibrium. We now prove that there is indeed abstention in equilibrium; this is done by showing that $L_{Q \rightarrow \emptyset}>L_{\emptyset \rightarrow A}$ in equilibrium which is indeed the same condition for the sets $\mathcal{W} \mathcal{S}^{A}, \mathcal{W} \mathcal{S}^{Q}$ and $\mathcal{A}$ to be non empty.

To prove that there is abstention in equilibrium, and hence the set of weak supporters and abstainers are not empty we first prove the following Lemma

Lemma $4 \operatorname{Let} T(y ; h)=\sum_{k=0}^{\left\lfloor\frac{n}{2}-1\right\rfloor} \frac{(n-1) !}{k ! h !(n-1-k-h) !}(y)^{k} ; \frac{T(k+1)}{T(k)}$ is increasing in $y$. 
Proof. Using the definition of $T(y ; h)$ we have that

$$
\frac{T(y ; k+1)}{T(y ; k)}=(n+1) \frac{1+\sum_{k=1}^{\left\lfloor\frac{n}{2}-1\right\rfloor} \frac{(n-1) !}{k !(k+1) !(n-1-2 k) !}(y)^{k}}{1+\sum_{k=1}^{\left\lfloor\frac{n}{2}-1\right\rfloor} \frac{(n-1) !}{k ! k !(n-1-2 k) !}(y)^{k}}-2
$$

Taking derivatives with respect to y leads, after some algebra to

$$
\begin{aligned}
&\left(\sum_{k=1}^{\left\lfloor\frac{n}{2}-1\right\rfloor} \frac{(n-1) !}{(k-1) !(k+1) !(n-1-2 k) !}(y)^{k}\right)\left(\sum_{k=1}^{\left\lfloor\frac{n}{2}-1\right\rfloor} \frac{(n-1) !}{k !(k+1) !(n-1-2 k) !}(k+1)(y)^{k}\right) \\
& \frac{d\left(\frac{T(y ; k+1)}{T(y ; k)}\right)}{d y} \leq \frac{(n+1)}{y} \frac{-\left(\sum_{k=1}^{\left\lfloor\frac{n}{2}-1\right\rfloor} \frac{(n-1) !}{k !(k+1) !(n-1-2 k) !}(y)^{k}\right)\left(\sum_{k=1}^{\left\lfloor\frac{n}{2}-1\right\rfloor} \frac{(n-1) !}{k !(k+1) !(n-1-2 k) !} k(k+1)(y)^{k}\right)}{\left(1+\sum_{k=1}^{\left\lfloor\frac{n}{2}-1\right\rfloor} \frac{(n-1) !}{k ! k !(n-1-2 k) !}(y)^{k}\right)^{2}}
\end{aligned}
$$

so it is sufficient to prove the result that

$$
0 \geq \sum_{j=1}^{\left\lfloor\frac{n}{2}-1\right\rfloor} \sum_{k=1}^{\left\lfloor\frac{n}{2}-1\right\rfloor} \frac{(n-1) !}{k !(k+1) !(n-1-2 k) !} \frac{(n-1) !}{j !(j+1) !(n-1-2 j) !}(k-j) j(y)^{j+k}
$$

where we have replaced the index $k$ for $j$ appropriately. The result follows by observing that

$$
\begin{aligned}
& 2 \sum_{k=1}^{\left\lfloor\frac{n}{2}-1\right\rfloor} \sum_{j=1}^{\left\lfloor\frac{n}{2}-1\right\rfloor} \frac{(n-1) !}{k !(k+1) !(n-1-2 k) !} \frac{(n-1) !}{j !(j+1) !(n-1-2 j) !}(k-j) j(y)^{j+k} \\
& =-\sum_{k=1}^{\left\lfloor\frac{n}{2}-1\right\rfloor} \sum_{j=k}^{\left\lfloor\frac{n}{2}-1\right\rfloor} \frac{(n-1) !}{k !(k+1) !(n-1-2 k) !} \frac{(n-1) !}{j !(j+1) !(n-1-2 j) !}(k-j)^{2}(y)^{j+k}
\end{aligned}
$$

The next Corollary follows from the condition (13) and presents sufficient conditions from abstention that are easier to check:

Corollary 1 There is abstention for some positive mass of types iff

$$
\frac{\operatorname{Pr}(A \mid a)}{\operatorname{Pr}(Q \mid a)}>1>\frac{\operatorname{Pr}(A \mid q)}{\operatorname{Pr}(Q \mid q)}
$$


Proof. Let $\left(x_{1}^{\omega}, x_{2}^{\omega}\right) \in X_{1}$ and we can define for $l \in\left\{x_{1}^{\omega}, x_{2}^{\omega}\right\}$

$$
\begin{aligned}
& \frac{\tau_{1}(\omega)}{\left(1-\left(x_{1}^{\omega}+x_{2}^{\omega}\right)\right)^{n-1}} \equiv \sum_{k=0}^{\left\lfloor\frac{n-1}{2}\right\rfloor} \frac{(n-1) !}{k ! k !(n-1-2 k) !}\left(\frac{x_{1}^{\omega}}{\left(1-\left(x_{1}^{\omega}+x_{2}^{\omega}\right)\right)} \frac{x_{2}^{\omega}}{\left(1-\left(x_{1}^{\omega}+x_{2}^{\omega}\right)\right)}\right)^{k} \\
& \frac{\tau_{2}(l, \omega)}{\left(1-\left(x_{1}^{\omega}+x_{2}^{\omega}\right)\right)^{n-1}} \equiv l \sum_{k=0}^{\left\lfloor\frac{n}{2}-1\right\rfloor} \frac{(n-1) !}{k !(k+1) !(n-2 k-2) !}\left(\frac{x_{1}^{\omega}}{\left(1-\left(x_{1}^{\omega}+x_{2}^{\omega}\right)\right)} \frac{x_{2}^{\omega}}{\left(1-\left(x_{1}^{\omega}+x_{2}^{\omega}\right)\right)}\right)^{k}
\end{aligned}
$$

where $\lfloor T\rfloor$ is the biggest integer smaller than $T$; note that $\tau_{1}(\omega)$ only depends on the state while $\tau_{2}(l, \omega)$ also depends on $l$. Replacing $x_{1}^{\omega}$ with $\operatorname{Pr}(A \mid \omega)$ and $x_{2}^{\omega}$ with $\operatorname{Pr}(Q \mid \omega)$ as defined in (1), $\tau_{1}(\omega)$ is the situation where $A$ and $Q$ are tied in state $\omega$ and $\tau_{2}(\operatorname{Pr}(A \mid \omega), \omega)$ $\left(\tau_{2}(\operatorname{Pr}(Q \mid \omega), \omega)\right)$ is the probability that candidate $A(Q)$ has an advantage of 1 vote in state $\omega$ and we have that

$$
\begin{aligned}
\operatorname{Pr}\left(\text { piv }_{A} \mid \omega\right) & =\frac{\tau_{2}(\operatorname{Pr}(Q \mid \omega), \omega)+\tau_{1}(\omega)}{2} \\
\operatorname{Pr}\left(\text { piv }_{Q} \cup \operatorname{piv}_{A} \mid \omega\right) & =\operatorname{Pr}\left(\text { piv }_{A} \mid \omega\right)+\frac{\tau_{2}(\operatorname{Pr}(A \mid \omega), \omega)+\tau_{1}(\omega)}{2}
\end{aligned}
$$

so $L_{Q \rightarrow A}>L_{\emptyset \rightarrow A}$ (see the discussion before condition (8)) is equivalent to

$$
\frac{\tau_{2}(\operatorname{Pr}(A \mid a), a)-\tau_{2}(\operatorname{Pr}(Q \mid a), a)}{\tau_{1}(a)+\tau_{2}(\operatorname{Pr}(Q \mid a), a)}>\frac{\tau_{2}(\operatorname{Pr}(A \mid q), q)-\tau_{2}(\operatorname{Pr}(Q \mid q), q)}{\tau_{1}(q)+\tau_{2}(\operatorname{Pr}(Q \mid q), q)}
$$

so it is sufficient if $\frac{\tau_{2}(\operatorname{Pr}(A \mid a), a)}{\tau_{2}(\operatorname{Pr}(Q \mid a), a)}>1>\frac{\tau_{2}(\operatorname{Pr}(A \mid q), q)}{\tau_{2}(\operatorname{Pr}(Q \mid q), q)}$. Using (33) we have that condition (32) is equivalent to condition (13) and it is then necessary for abstention.

Now we need to prove that $L_{Q \rightarrow A}>L_{\emptyset \rightarrow A}$ is actually sufficient for abstention but this was indeed proven when describing the set $\mathcal{W S}^{A}, \mathcal{W S}^{Q}$, and $\mathcal{A}$ which are non empty when $L_{Q \rightarrow \emptyset}>L_{\emptyset \rightarrow A}$.

Now we are ready to show that there is abstention. We are going to prove by contradiction so assume that there is no endogenous abstention because $L_{Q \rightarrow \emptyset} \leq L_{\emptyset \rightarrow A}$. For any fixed $N$, Proposition 1 implies that only three sets of voters may be not empty: strong supporters and independents so we only need the cutoff functions $g^{(A A)=(Q A)}\left(\theta_{a}\right)$ and $g^{(Q Q)=(Q A)}\left(\theta_{a}\right)$ to separate the set of types. The set of strong supporters is defined as

$$
\begin{aligned}
& \mathcal{S S}^{A} \equiv\left\{\theta \in[0,1]^{2}: \theta_{q} \leq g^{(A A)=(Q A)}\left(\theta_{a}\right)\right\} \\
& \mathcal{S S}^{Q} \equiv\left\{\theta \in[0,1]^{2}: \theta_{q} \geq g^{(Q Q)=(Q A)}\left(\theta_{a}\right)\right\}
\end{aligned}
$$

Using (2) and (3) and then definition of $\mathcal{L}(x)$ we have that

$$
\mathcal{L}\left(P^{Q A}\left(g^{(Q Q)=(Q A)}\left(\theta_{a}\right), \theta_{a}\right)\right)=g^{(Q Q)=(Q A)}\left(\theta_{a}\right) \times \frac{\operatorname{Pr}\left(\operatorname{piv}_{Q} \cup p i v_{A} \mid q\right)}{2}
$$

which implies that $g^{(Q Q)=(Q A)}\left(\theta_{a}\right)$ is strictly convex and that $g^{(A A)=(Q A)}(0)=g^{(Q Q)=(Q A)}(0)$. Since (24) implies that $g^{(A A)=(Q A)}\left(\theta_{a}\right)$ is strictly concave it follows that for any $\theta_{a}>0$, 
$g^{(Q Q)=(Q A)}\left(\theta_{a}\right)>g^{(A A)=(Q A)}\left(\theta_{a}\right)$. Therefore, the union of the sets $A$ strong supporters and for $Q$ does not cover the whole set of types which implies that $\mathcal{I} \neq \emptyset$ and there is information acquisition in equilibrium. Since abstention is exogenous and the same in both states we must have that

$$
\begin{aligned}
& \operatorname{Pr}(A \mid a)>\operatorname{Pr}(A \mid q) \\
& \operatorname{Pr}(Q \mid a)<\operatorname{Pr}(Q \mid q)
\end{aligned}
$$

Since there is no endogenous abstention, Corollary 1 and (35), imply one of the next two possibilities: 1) $\frac{\operatorname{Pr}(A \mid a)}{\operatorname{Pr}(Q \mid a)} \leq 1$, or 2) $\frac{\operatorname{Pr}(A \mid q)}{\operatorname{Pr}(Q \mid q)} \geq 1$.

Case 1:

Since $\frac{\operatorname{Pr}(A \mid a)}{\operatorname{Pr}(Q \mid a)} \leq 1$ we have the following order of probabilities of voting for candidates given the state

$$
\operatorname{Pr}(A \mid q)<\operatorname{Pr}(A \mid a) \leq \operatorname{Pr}(Q \mid a)<\operatorname{Pr}(Q \mid q)
$$

and also that

$$
\begin{aligned}
\frac{\operatorname{Pr}(A \mid q)}{\operatorname{Pr}(Q \mid q)+\operatorname{Pr}(A \mid q)} & <\frac{\operatorname{Pr}(A \mid a)}{\operatorname{Pr}(Q \mid a)+\operatorname{Pr}(A \mid a)} \leq \frac{1}{2} \\
\frac{1}{2} & \leq \frac{\operatorname{Pr}(Q \mid a)}{\operatorname{Pr}(Q \mid a)+\operatorname{Pr}(A \mid a)}<\frac{\operatorname{Pr}(Q \mid q)}{\operatorname{Pr}(Q \mid q)+\operatorname{Pr}(A \mid q)}
\end{aligned}
$$

since there is no endogenous abstention and abstention does not depend on the state. Note that the function $f_{1}(x)=x(1-x)$ is single peaked which implies that

$$
f_{1}\left(\frac{\operatorname{Pr}(A \mid a)}{\operatorname{Pr}(Q \mid a)+\operatorname{Pr}(A \mid a)}\right)>f_{1}\left(\frac{\operatorname{Pr}(A \mid q)}{\operatorname{Pr}(Q \mid q)+\operatorname{Pr}(A \mid q)}\right)
$$

so we must have that

$$
\begin{aligned}
& \left(\frac{\operatorname{Pr}(A \mid a)}{(1-(\operatorname{Pr}(A \mid a)+\operatorname{Pr}(Q \mid a)))} \frac{\operatorname{Pr}(Q \mid a)}{(1-(\operatorname{Pr}(A \mid a)+\operatorname{Pr}(Q \mid a)))}\right)^{k} \\
& =\left(f_{1}\left(\frac{\operatorname{Pr}(A \mid a)}{\operatorname{Pr}(Q \mid a)+\operatorname{Pr}(A \mid a)}\right)\right)^{k}\left(\frac{(\operatorname{Pr}(A \mid a)+\operatorname{Pr}(Q \mid a))}{(1-(\operatorname{Pr}(A \mid a)+\operatorname{Pr}(Q \mid a)))}\right)^{2 k} \\
& >\left(f_{1}\left(\frac{\operatorname{Pr}(A \mid q)}{\operatorname{Pr}(Q \mid q)+\operatorname{Pr}(A \mid q)}\right)\right)^{k}\left(\frac{(\operatorname{Pr}(A \mid q)+\operatorname{Pr}(Q \mid q))}{(1-(\operatorname{Pr}(A \mid q)+\operatorname{Pr}(Q \mid q)))}\right)^{2 k}
\end{aligned}
$$

where the second line follows because $\operatorname{Pr}(Q \mid q)+\operatorname{Pr}(A \mid q)=\operatorname{Pr}(Q \mid a)+\operatorname{Pr}(A \mid a)=$ $1-\alpha+\alpha\left(\xi_{A}+\xi_{Q}\right)$, the third line follows by (37), and the last line follows since abstention is constant across states (only partisan voters abstain). Using (33) from Proposition 3 with $\operatorname{Pr}(A \mid \omega)$ instead of $x_{1}^{\omega}$ and $\operatorname{Pr}(Q \mid \omega)$ instead of $x_{2}^{\omega}$ we must have that $\tau_{1}(a)>\tau_{1}(q)$ and $\tau_{2}(l, a)>\tau_{2}(l, q)$.

Recalling Corollary 1 we have that is necessary and sufficient for abstention that the 
following inequality holds:

$$
\frac{\tau_{1}(a)+\operatorname{Pr}(A \mid a) \widetilde{\tau}_{2}(a)}{\tau_{1}(a)+\operatorname{Pr}(Q \mid a) \widetilde{\tau}_{2}(a)}>\frac{\tau_{1}(q)+\operatorname{Pr}(A \mid q) \widetilde{\tau}_{2}(q)}{\tau_{1}(q)+\operatorname{Pr}(Q \mid q) \widetilde{\tau}_{2}(q)}
$$

where

$$
\frac{\widetilde{\tau}_{2}(\omega)}{\left(1-\left(x_{1}^{\omega}+x_{2}^{\omega}\right)\right)^{n-1}}=\frac{\tau_{2}(l, \omega)}{\left(1-\left(x_{1}^{\omega}+x_{2}^{\omega}\right)\right)^{n-1}} \frac{1}{l}
$$

Note that we can manipulate the sufficient condition (38) to get $\frac{\operatorname{Pr}(Q \mid a)-\operatorname{Pr}(A \mid a)}{\frac{\tau_{1}(a)}{\tau_{2}(a)}+\operatorname{Pr}(Q \mid a)}<\frac{\operatorname{Pr}(Q \mid q)-\operatorname{Pr}(A \mid q)}{\frac{\tau_{1}(q)}{\tau_{2}(q)}+\operatorname{Pr}(Q \mid q)}$ or

$$
\begin{aligned}
& \frac{\tau_{1}(q)}{\widetilde{\tau}_{2}(q)}(\operatorname{Pr}(Q \mid a)-\operatorname{Pr}(A \mid a))+\operatorname{Pr}(Q \mid a) \operatorname{Pr}(A \mid q) \\
& <\frac{\tau_{1}(a)}{\widetilde{\tau}_{2}(a)}(\operatorname{Pr}(Q \mid q)-\operatorname{Pr}(A \mid q))+\operatorname{Pr}(Q \mid q) \operatorname{Pr}(A \mid a)
\end{aligned}
$$

Recalling that $0<\operatorname{Pr}(Q \mid a)-\operatorname{Pr}(A \mid a)<\operatorname{Pr}(Q \mid q)-\operatorname{Pr}(A \mid q)$ by $(36)$, and $\operatorname{Pr}(Q \mid q) \operatorname{Pr}(A \mid a)>$ $\operatorname{Pr}(Q \mid a) \operatorname{Pr}(A \mid q)$ by the fact that there is information acquisition, if $\frac{\tau_{1}(a)}{\widetilde{\tau}_{2}(a)} \geq \frac{\tau_{1}(q)}{\widetilde{\tau}_{2}(q)}$ we have that (38) hold.

Recalling the definition of $T(y ; h)$ in Lemma 4 we have that $\frac{T\left(\frac{\operatorname{Pr}(A \mid \omega) \operatorname{Pr}(Q \mid \omega)}{(1-(\operatorname{rr}(A \mid \omega)+\operatorname{Pr}(Q \mid \omega)))^{2}} ; k+1\right)}{T\left(\frac{\operatorname{Pr}(A \mid \omega) \operatorname{Pr}(Q \mid \omega)}{(1-(\operatorname{Pr}(A \mid \omega)+\operatorname{Pr}(Q \mid \omega)))^{2}} ; k\right)}=$ $\frac{\widetilde{\tau}_{2}(\omega)}{\tau_{1}(\omega)}$. Using the result in $(37)$ we have that Lemma 4 gives that $\frac{\widetilde{\tau}_{2}(a)}{\tau_{1}(a)}<\frac{\widetilde{\tau}_{2}(q)}{\tau_{1}(q)}$ since $\frac{\operatorname{Pr}(A \mid a) \operatorname{Pr}(Q \mid a)}{(1-(\operatorname{Pr}(A \mid a)+\operatorname{Pr}(Q \mid a)))^{2}}>$ $\frac{\operatorname{Pr}(A \mid q) \operatorname{Pr}(Q \mid q)}{(1-(\operatorname{Pr}(A \mid q)+\operatorname{Pr}(Q \mid q)))^{2}}$ which leads to a contradiction and $\frac{\operatorname{Pr}(A \mid a)}{\operatorname{Pr}(Q \mid a)} \leq 1$ is not possible.

Case 2:

The case $\frac{\operatorname{Pr}(A \mid q)}{\operatorname{Pr}(Q \mid q)} \geq 1$ uses that it must be that

$$
\operatorname{Pr}(Q \mid a)<\operatorname{Pr}(Q \mid q) \leq \operatorname{Pr}(A \mid q)<\operatorname{Pr}(A \mid a)
$$

and (37) implies that $\tau_{1}(a)<\tau_{1}(q)$ and $\tau_{2}(l, a)<\tau_{2}(l, q)$. Since (38) can be written as

$$
\begin{gathered}
\frac{\operatorname{Pr}(A \mid a)-\operatorname{Pr}(Q \mid a)}{\frac{\tau_{1}(a)}{\widetilde{\tau}_{2}(a)}+\operatorname{Pr}(Q \mid a)}>\frac{\operatorname{Pr}(A \mid q)-\operatorname{Pr}(Q \mid q)}{\frac{\tau_{1}(q)}{\widetilde{\tau}_{2}(q)}+\operatorname{Pr}(Q \mid q)} \\
\left(\frac{\tau_{1}(q)}{\widetilde{\tau}_{2}(q)}+\operatorname{Pr}(Q \mid q)\right)(\operatorname{Pr}(A \mid a)-\operatorname{Pr}(Q \mid a))>\left(\frac{\tau_{1}(a)}{\widetilde{\tau}_{2}(a)}+\operatorname{Pr}(Q \mid a)\right)(\operatorname{Pr}(A \mid q)-\operatorname{Pr}(Q \mid q))
\end{gathered}
$$

and since $\operatorname{Pr}(A \mid a)-\operatorname{Pr}(Q \mid a)>\operatorname{Pr}(A \mid q)-\operatorname{Pr}(Q \mid q) \geq 0$ it is sufficient if $\frac{\tau_{1}(q)}{\widetilde{\tau}_{2}(q)}>\frac{\tau_{1}(a)}{\widetilde{\tau}_{2}(a)}$. Using again that $\frac{T\left(\frac{\operatorname{Pr}(A \mid \omega) \operatorname{Pr}(Q \mid \omega)}{(1-(\operatorname{Pr}(A \mid \omega)+\operatorname{Pr}(Q \mid \omega)))^{2}} ; k+1\right)}{T\left(\frac{\operatorname{Pr}(A \mid \omega) \operatorname{Pr}(Q \mid \omega)}{(1-(\operatorname{Pr}(A \mid \omega)+\operatorname{Pr}(Q \mid \omega)))^{2}} ; k\right)}=\frac{\widetilde{\tau}_{2}(\omega)}{\tau_{1}(\omega)}$, Lemma 4 gives that $\frac{\widetilde{\tau}_{2}(a)}{\tau_{1}(a)}>\frac{\widetilde{\tau}_{2}(q)}{\tau_{1}(q)}$ since now (37) and (39) implies that $\frac{\operatorname{Pr}(A \mid a) \operatorname{Pr}(Q \mid a)}{(1-(\operatorname{Pr}(A \mid a)+\operatorname{Pr}(Q \mid a)))^{2}}<\frac{\operatorname{Pr}(A \mid q) \operatorname{Pr}(Q \mid q)}{(1-(\operatorname{Pr}(A \mid q)+\operatorname{Pr}(Q \mid q)))^{2}}$. 
Proof of Proposition 3 Let $\phi=\frac{\left(1-\left(\xi_{A}+\xi_{Q}\right) \alpha\right)^{n-1}}{2}$ and define the spaces

$$
\begin{aligned}
X_{1} & \equiv\left\{(x, y) \in\left[\xi_{A} \alpha, 1-\left(\xi_{\emptyset}+\xi_{Q}\right) \alpha\right] \times\left[\xi_{Q} \alpha, 1-\left(\xi_{\emptyset}+\xi_{A}\right) \alpha\right]\right\} \\
X_{2}(\phi) & \equiv\left\{(x, y, v, z) \in[\phi, 1]^{2} \times[\phi, 1]^{2}: x+\phi \leq v, y+\phi \leq z\right\}
\end{aligned}
$$

Let $\left(x_{1}^{\omega}, x_{2}^{\omega}\right) \in X_{1}$ be a generic element of the set $X_{1}$ if we let $\omega=a(\omega=q)$ so $\left(x_{1}^{\omega}, x_{2}^{\omega}\right)$ plays the role of the probabilities a random non partisan voter supports $A(Q)$ in different states. Let $\left(y_{\emptyset}^{a}, y_{\emptyset}^{q}, y_{Q}^{a}, y_{Q}^{q}\right)$ by a generic element of the space $X_{2}(\phi)$ so $y_{\emptyset}^{\omega}$ plays the role of $\operatorname{Pr}\left(\right.$ piv $\left._{A} \mid \omega\right)$ and $y_{Q}^{\omega}$ plays the role of $\operatorname{Pr}\left(\right.$ piv $_{Q} \cup$ piv $\left._{A} \mid \omega\right)$ for $\omega \in\{a, q\}$.

Let $p^{i}:[0,1]^{2} \times X_{2}(\phi) \rightarrow\left[\frac{1}{2}, 1-\eta\right], i=1,2,3$ be implicitly defined by $C^{\prime}\left(p^{1}\right)=\frac{\theta_{a} y_{\emptyset}^{a}+\theta_{q} y_{\emptyset}^{q}}{2}$, $C^{\prime}\left(p^{2}\right)=\frac{\theta_{a} y_{Q}^{a}+\theta_{q} y_{Q}^{q}}{2}$, and $C^{\prime}\left(p^{3}\right)=\frac{\theta_{a}\left(y_{Q}^{a}-y_{\emptyset}^{a}\right)+\theta_{q}\left(y_{Q}^{q}-y_{\emptyset}^{q}\right)}{2}$, and let $\eta$ be such that $C^{\prime}(1-\eta)>1$. So $p^{1}$ plays the role of $P^{\emptyset A}, p^{2}$ plays the role of $P^{Q A}$ and $p^{3}$ plays the role of $P^{Q \emptyset}$ as defined in (11). Now consider an element $\left(y_{\emptyset}^{a}, y_{\emptyset}^{q}, y_{Q}^{a}, y_{Q}^{q}\right) \in X_{2}(\phi)$ and using $\left(p^{1}, p^{2}, p^{3}\right)$, we can define the cutoff functions used in the characterization of equilibrium . Therefore, the sets of strong and weak supporters, independents and abstainers are well defined. Using Proposition 1 we have that $P\left(X^{\omega}\right)$, the probability of a vote for $X \in\{Q, A\}$ in state $\omega \in\{q, a\}$, is

$$
\begin{aligned}
\operatorname{Pr}\left(A^{a}\right) & \equiv \int_{\theta \in \mathcal{W} \mathcal{S}^{A}} p^{1}(\theta) d F(\theta)+\int_{\theta \in \mathcal{S S}^{A}} d F(\theta)+\int_{\theta \in \mathcal{I}} p^{2}(\theta) d F(\theta) \\
\operatorname{Pr}\left(A^{q}\right) & \equiv \int_{\theta \in \mathcal{W S}^{A}}\left(1-p^{1}(\theta)\right) d F(\theta)+\int_{\theta \in \mathcal{S} \mathcal{S}^{A}} d F(\theta)+\int_{\theta \in \mathcal{I}}\left(1-p^{2}(\theta)\right) d F(\theta) \\
\operatorname{Pr}\left(Q^{q}\right) & \int_{\theta \in \mathcal{W} \mathcal{S}^{Q}} p^{3}(\theta) d F(\theta)+\int_{\theta \in \mathcal{S S}^{Q}} d F(\theta)+\int_{\theta \in \mathcal{I}} p^{2}(\theta) d F(\theta) \\
\operatorname{Pr}\left(Q^{a}\right) & \equiv \int_{\theta \in \mathcal{W} \mathcal{S}^{Q}}\left(1-p^{3}(\theta)\right) d F(\theta)+\int_{\theta \in \mathcal{S} \mathcal{S}^{Q}} d F(\theta)+\int_{\theta \in \mathcal{I}}\left(1-p^{2}(\theta)\right) d F(\theta)
\end{aligned}
$$

For functions $\left(p^{1}, p^{2}, p^{3}\right)$ and $\left(y_{\emptyset}^{a}, y_{\emptyset}^{q}, y_{Q}^{a}, y_{Q}^{q}\right) \in X_{2}(\phi)$ we define the functions $G_{X}^{\omega}$ : $X_{2}(\phi) \rightarrow X_{1}$ for $X=A, Q$ such that

$$
\begin{aligned}
& G_{A}^{\omega}\left(y_{\emptyset}^{a}, y_{\emptyset}^{q}, y_{Q}^{a}, y_{Q}^{q}\right) \equiv \xi_{A} \alpha+(1-\alpha) \operatorname{Pr}\left(A^{\omega}\right) \\
& G_{Q}^{\omega}\left(y_{\emptyset}^{a}, y_{\emptyset}^{q}, y_{Q}^{a}, y_{Q}^{q}\right) \equiv \xi_{Q} \alpha+(1-\alpha) \operatorname{Pr}\left(Q^{\omega}\right)
\end{aligned}
$$

Let $\left(x_{1}^{\omega}, x_{2}^{\omega}\right) \in X_{1}$ and using (33) we have that $\tau_{1}(\omega)$ is the situation where $A$ and $Q$ are tied in state $\omega$ and $\tau_{2}\left(x_{1}^{\omega}, \omega\right)\left(\tau_{2}\left(x_{2}^{\omega}, \omega\right)\right)$ is the probability that candidate $A(Q)$ has an advantage of 1 vote in state $\omega$. Recalling $\operatorname{Pr}\left(\right.$ piv $\left._{A} \mid \omega\right)$ and $\operatorname{Pr}\left(\right.$ piv $_{Q} \cup$ piv $\left._{A} \mid \omega\right)$ we define the function $K^{\omega}: X_{1} \times X_{1} \rightarrow X_{2}(\phi)$ as $K_{1}^{\omega}\left(x_{1}^{a}, x_{2}^{a}, x_{1}^{q}, x_{2}^{q}\right)=\frac{\tau_{2}\left(x_{2}^{\omega}, \omega\right)+\tau_{1}(\omega)}{2}$ and $K_{2}^{\omega}\left(x_{1}^{a}, x_{2}^{a}, x_{1}^{q}, x_{2}^{q}\right)=$ $\tau_{1}(\omega)+\frac{\tau_{2}\left(x_{1}^{\omega}, \omega\right)+\tau_{2}\left(x_{2}^{\omega}, \omega\right)}{2}$ so $K_{1}^{\omega}$ gives $\operatorname{Pr}\left(\right.$ piv $\left._{A} \mid \omega\right)$ and $K_{2}^{\omega}$ gives $\operatorname{Pr}\left(\right.$ piv $_{Q} \cup$ piv $\left.A \mid \omega\right) .{ }^{22}$

\footnotetext{
${ }^{22}$ Imagine that $x_{2}^{\omega}\left(x_{1}^{\omega}\right)$ is the probability of a vote in favor of $Q(A)$ in state $\omega$ and imagine a voter
} 
Now we have all the elements to show that an equilibrium actually exists. Take an arbitrary element of $\mathcal{S} \equiv\left(X_{1}\right)^{2} \times X_{2}(\phi)$, define the function $\Gamma: \mathcal{S} \rightarrow \mathcal{S}$ such that $\Gamma \equiv$ $\left\{G_{A}^{a}, G_{Q}^{a}, G_{A}^{q}, G_{Q}^{q}, K_{1}^{\omega}, K_{2}^{\omega}\right\}$, where the components are defined as above. We are going to show first that actually $\Gamma$ is a continuous function.

For continuity of $\left(G_{A}^{a}, G_{Q}^{a}, G_{A}^{q}, G_{Q}^{q}\right)$ we first observe that all the cutoff functions that determine the types (weak and strong supporters, abstainers and independents), are well defined and continuous for $\left(y_{\emptyset}^{a}, y_{\emptyset}^{q}, y_{Q}^{a}, y_{Q}^{q}\right)$ and $\left(p^{1}, p^{2}, p^{3}\right)$ as defined above. Therefore $\operatorname{Pr}\left(A^{\omega}\right)$ and $\operatorname{Pr}\left(Q^{\omega}\right)$ are continuous on $\left(y_{\emptyset}^{a}, y_{\emptyset}^{q}, y_{Q}^{a}, y_{Q}^{q}\right)$ when we consider that $\left(p^{1}, p^{2}, p^{3}\right)$ are also continuous and well defined for $y_{\emptyset}^{\omega} \in[\phi, 1], y_{Q}^{\omega} \in[\phi, 1]$. The fact that $K$ is continuous in $\left(x_{1}^{a}, x_{2}^{a}, x_{1}^{q}, x_{2}^{q}\right)$ follows trivially by continuity of $\tau_{2}(l, \omega)$ and $\tau_{1}(\omega)$ in $\left(x_{1}^{a}, x_{2}^{a}, x_{1}^{q}, x_{2}^{q}\right) . X_{1}$ and $X_{2}(\phi)$ are convex and compact, so Brouwer's fixed point theorem holds and there is some $x \in \mathcal{S}$ such that $\Gamma(x)=x$.

Proof of Proposition 2 Remark 1, Remark 2, and Remark 5 in the Appendix show that for $\theta_{a}>0$ but small, the cutoff functions are ordered according to

$$
g^{(Q Q)=(Q \emptyset)}\left(\theta_{a}\right)>g^{(Q \emptyset)=(\emptyset \emptyset)}\left(\theta_{a}\right)>g^{(\emptyset A)=(\emptyset \emptyset)}\left(\theta_{a}\right)>g^{(A A)=(\emptyset A)}\left(\theta_{a}\right)
$$

which gives the result.

Proof of Proposition 4 The first part follows by noting that $\operatorname{Pr}\left(\right.$ piv $_{Q} \cup$ piv $\left.A \mid \omega\right) \rightarrow 0$ when $n \rightarrow \infty$. Using that no information is collected in the limit we have that $\operatorname{Pr}(A \mid \omega)=$ $\operatorname{Pr}(A)$ and $\operatorname{Pr}(Q \mid \omega)=\operatorname{Pr}(Q)$ which implies that inequality (32) in Corollary 1 does not hold and abstention by non partisans voters has limit measure 0 .

Proof of Lemma 3 Since the argument in Proposition 1 does not depend on the fact that there is no endogenous abstention but just on (35) we have that $\frac{\operatorname{Pr}(A \mid q)}{\operatorname{Pr}(Q \mid q)}<1<\frac{\operatorname{Pr}(A \mid a)}{\operatorname{Pr}(Q \mid a)}$. Using now that in the limit almost no information is used and $\operatorname{Pr}(A \mid a) \rightarrow \operatorname{Pr}(A \mid q)$ and $\operatorname{Pr}(Q \mid a) \rightarrow \operatorname{Pr}(Q \mid q)$ we have for sufficiently large $N$ it must be that $1 \approx \frac{\operatorname{Pr}(A \mid q)}{\operatorname{Pr}(Q \mid q)}$ so in the limit

$$
\alpha \xi_{Q}+\int_{\theta \in \mathcal{S S}^{Q}} f(\theta) d \theta \approx \alpha \xi_{A}+\int_{\theta \in \mathcal{S} \mathcal{S}^{A}} f(\theta) d \theta
$$

which gives the result.

considering switching her vote from $\emptyset$ to $A$. Then $\frac{\tau_{1}(\omega)}{2}$ is the increment in the probability of $A$ winning because a tie is broken and $\frac{\tau_{2}\left(x_{2}^{\omega}, \omega\right)}{2}$ is increment in the probability of $A$ winning because a tie is created. 